\title{
Analyse des textes juridiques et de la littérature sur les systèmes de tenure vivante à Madagascar
}

Rebecca McLain

Patrick Ranjatson

Tasha Heidenrich

Jean Mananga Rakotonirina

Andrisoa Richard Fabrico Nomenjanahary

Ny Tolotra Ramboatsitoharana Razafimbelo
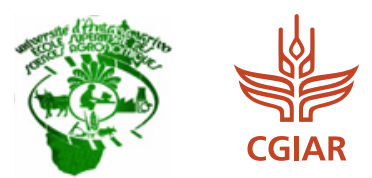

Document de travail 281

\section{Analyse des textes juridiques et de la littérature sur les systèmes de tenure vivante à Madagascar}

Rebecca McLain

CIFOR

Patrick Ranjatson

ESSA-Forêts

Tasha Heidenrich

Consultante indépendante

Jean Mananga Rakotonirina

Consultante indépendante

Andrisoa Richard Fabrico Nomenjanahary

ESSA-Forêts

Ny Tolotra Ramboatsitoharana Razafimbelo, ESSA-Forêts 
Document de travail 281

(C) 2021 Centre de recherche forestière internationale (CIFOR)

(c) (i) Le contenu de cette publication est soumis à une licence des Creative Commons Attribution 4.0 International (CC BY 4.0), http://creativecommons.org/licenses/by/4.0//

DOI: $10.17528 /$ cifor $/ 008388$

McLain R, Ranjatson P, Heidenrich T, Rakotonirina JM, Nomenjanahary ARF et Razafimbelo NTR. 2021. Analyse des textes juridiques et de la littérature sur les systèmes de tenure vivante à Madagascar. Document de travail 281. Bogor, Indonésie : CIFOR.

Traduction de : McLain R, Ranjatson P, Heidenrich T, Rakotonirina JM, Nomenjanahary ARF and Razafimbelo NTR. 2021. An exploratory review of legal texts and literature on living tenure systems in Madagascar. Working Paper 278. Bogor, Indonesia: CIFOR.

\section{CIFOR}

Jl. CIFOR, Situ Gede

Bogor Barat 16115

Indonésie

$\mathrm{T}+62(251) 8622-622$

$\mathrm{F}+62(251) 8622-100$

E cifor@cgiar.org

\section{cifor.org}

Nous tenons à remercier tous les donateurs qui ont soutenu cette recherche avec leurs contributions au Fonds du CGIAR. Pour une liste des donateurs au Fonds, s'il vous plaît voir : http://www.cgiar.org/our-funders/

Tous les points de vue figurant dans cet ouvrage sont ceux des auteurs. Ils ne représentent pas forcément les points de vue du CIFOR, des responsables de la rédaction, des institutions respectives des auteurs, des soutiens financiers ou des relecteurs. 


\section{Table des matières}

Sigles et acronymes

$\begin{array}{ll}\text { Remerciements } & \text { vi }\end{array}$

1 Introduction $\quad 1$

2 Les textes juridiques relatifs à la tenure foncière communautaire $\quad 2$

2.1 Aperçu des catégories de tenure foncière étatique et des divisions administratives 2

2.2 Les collectivités au niveau local 3

2.3 Les collectivités territoriales décentralisées 3

$\begin{array}{ll}2.4 \text { Le fokontany } & 5\end{array}$

$\begin{array}{ll}2.5 & \text { Le fokonolona }\end{array}$

2.6 Les groupes légalement constitués : Vondron'olona ifotony (communautés de base en malgache) 7

$\begin{array}{lll}2.7 & \text { La communauté et la tenure foncière } & 8\end{array}$

$\begin{array}{lll}2.8 \text { Les textes juridiques cités } & 9\end{array}$

3 Les grands axes de la gouvernance foncière au niveau local à Madagascar 11

$\begin{array}{lll}3.1 \text { Introduction } & 11\end{array}$

3.2 Le pluralisme juridique : à la croisée des systèmes étatiques et coutumiers en matière de tenure et de gouvernance $\quad 11$

3.3 La gouvernance locale traditionnelle $\quad 14$

3.4 Le Dina : lorsque le droit coutumier rencontre le droit écrit 19

$\begin{array}{lll}3.5 & \text { Les droits fonciers traditionnels } & 19\end{array}$

$\begin{array}{lll}3.6 & \text { Les droits fonciers des femmes } & 21\end{array}$

3.7 Les catégories locales d'utilisation des terres et les droits fonciers correspondants 23

3.8 Les principaux facteurs de changement dans l'utilisation des terres et les systèmes de tenure coutumière

3.9 Les principales lacunes présentes dans la recherche $\quad 32$

4 Conclusion $\quad 34$

$\begin{array}{ll}\text { References } & \mathbf{3 6}\end{array}$

\section{Table des figures, tableau et encadrés}

\section{Figures}

1 La structure de gouvernance parallèle à Madagascar 3

2 Les régions de Madagascar $\quad 12$

3 La localisation des études sélectionnées $\quad 13$

\section{Tableau}

1 Les groupes ethniques malgaches

\section{Encadrés}

1 Exemples de structures de gouvernance foncière 17

2 Dina à Ambodilaingo (Commune de Beforona) 19

3 Privatisation d'une ressource commune 26

4 Exemples de règles relatives aux forêts sacrées à Madagascar 29 


\section{Sigles et acronymes}

$\begin{array}{ll}\text { COBA } & \text { Communauté de base } \\ \text { CTD } & \text { Collectivité territoriale décentralisée } \\ \text { GCF } & \text { Gestion contractualisée des forêts } \\ \text { GELOSE } & \text { Gestion locale sécurisée } \\ \text { SFR } & \text { Sécurisation Foncière Relative } \\ \text { PAG } & \text { Plan d'aménagement et de gestion } \\ \text { IRD } & \text { Institut de Recherche pour le Développement } \\ \text { ORSTOM } & \text { Office de la Recherche Scientifique et Technique Outre-mer } \\ \text { VOI } & \text { Vondron'olona ifotony (Communauté de base en malgache) }\end{array}$

\section{Glossaire malgache}

ala
ala be
alafady
ala fano-hofa
ala kibory
ala mandiniky
ampanjaka
baiboho
dina
fady, faly
famahidana
fanjakana
fatidra
fiarana
fihavanana
filongoa

fokonolona; fokonolo fokontany

havana

karazaña

kija

kily

kitay telo an-dalana

kizo

lazo
Forêt

Grande forêt

Forêt taboue/sacrée

Bosquet de miel (Androy)

Parcelles de forêt avec des tombes (Androy)

Petite forêt dégradée (Sakalava)

Roi

Plaines avec inondation saisonnière

Règlements locaux, convention sociale

Tabou

Rituel de retournement des morts

Autorités de l'État

Rituel de mélange des sangs

Coopération

Solidarité

Cohésion sociale, mais englobe généralement la parenté essentiellement patrilinéaire

Conseil de village entériné par l'État et légitime au niveau local

Échelon administratif le plus bas de l'appareil étatique

Famille, relation

Groupe ethnique/tribu

Zone où l'on rassemble les zébus ${ }^{1}$ (pour brouter, s'abreuver, barboter, se reposer)

Tamarinier

Partage traditionnel des biens en cas de divorce (deux tiers reviennent au mari, un tiers à la femme)

Chemins pour le passage des zébus

Arbre coupé

1 Le zébu est une espèce ou sous-espèce de bovidé domestique originaire du sous-continent indien, caractérisé par une bosse graisseuse située sur les épaules, un fanon largement développé et parfois des oreilles tombantes. 

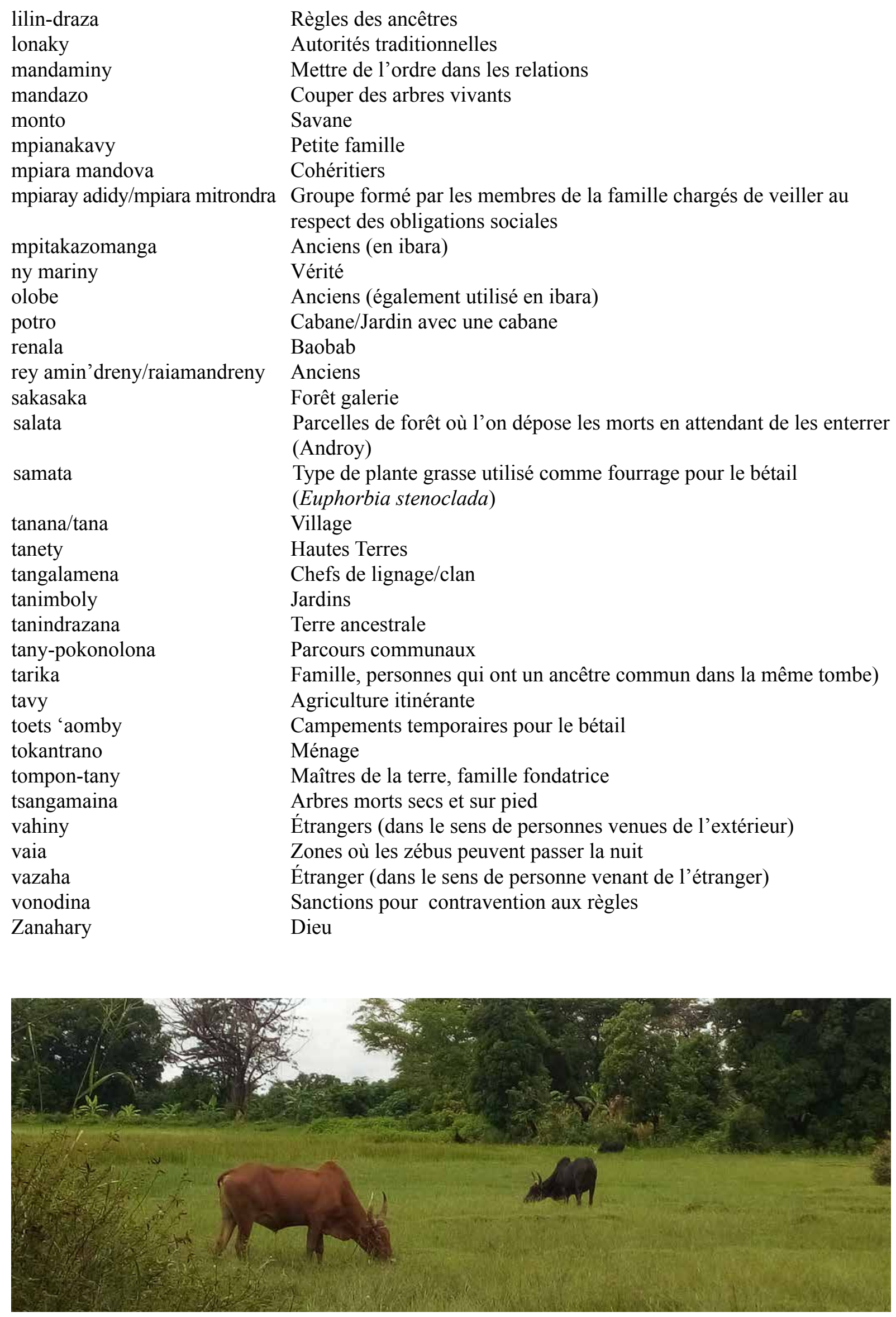

Le pâturage des zébus dans le nord-ouest de Madagascar.

Photo par Ny Tolotra Razafimbelo 


\section{Remerciements}

Ce travail a été financé à travers et entrepris dans le cadre des programmes de recherche du CGIAR sur les politiques, institutions et marchés (PIM), dirigé par l'Institut International de Recherche sur les Politiques Alimentaires (IFPRI) ; sur les Forêts, Arbres et Agroforesterie (FTA), dirigé par le CIFOR. Ce document de travail n'a pas été soumis aux procédures standards d'examen par les pairs de l'IFPRI. Les opinions exprimées ici appartiennent aux auteurs et ne reflètent pas nécessairement celles du CIFOR, du PIM, de l'IFPRI, du CGIAR ou des sponsors financiers.

Nous tenons à adresser nos remerciements à Andrisoa Richard Fabrico Nomenjanahary pour la création des cartes utilisées dans ce document de travail. Nous tenons également à exprimer notre gratitude à Nantenaina Fitiavana Jean Renaud Randrianasolo pour sa connaissance des systèmes de tenure foncière pastorale, ainsi qu'à Anne Larson pour son soutien intellectuel continu. 


\section{Introduction}

En 2018 et 2019, le Centre de recherche forestière internationale (CIFOR), en collaboration avec son partenaire malgache, l'École supérieure des sciences agronomiques (ESSA-Forêts) de l'Université d'Antananarivo, a mené un projet de recherche dans la région de Boeny, située dans le nord-ouest de Madagascar, afin de comprendre les éventuelles conséquences de la tenure foncière sur les efforts visant à soutenir un engagement massif dans la restauration des paysages forestiers (Ranjatson et al. 2019). D'après cette étude, il semble que le droit foncier actuellement en vigueur offre une sécurité foncière inadéquate pour les terres rurales qui sont détenues et gérées collectivement (McLain et al. 2019). Les modalités de détermination de l'entité ou des entités reconnue(s) juridiquement en tant que collectivité(s) (et disposant donc de la personnalité juridique qui leur permettrait de détenir et de gérer des droits fonciers) font actuellement l'objet d'un débat à Madagascar.

Les efforts bien intentionnés visant à fournir aux ruraux des certificats fonciers individuels (conformément la loi foncière de 2005) risquent de saper les droits collectifs sur les forêts, les pâturages ainsi que sur les autres espaces communs (McLain et al. 2019). Ces espaces communs sont précisément les zones les plus susceptibles d'être ciblées pour la restauration des paysages forestiers. Cependant, le manque d'informations sur les systèmes de tenure coutumière vivante (qui régissent en pratique l'utilisation de ces communs) entrave les efforts destinés à identifier le ou les niveaux auxquels les collectivités chargées d'attribuer les terres et de faire valoir les revendications foncières devraient recevoir une reconnaissance juridique. Nous décrivons ces systèmes de tenure coutumière comme étant « vivants » pour souligner qu'il s'agit de systèmes coutumiers dynamiques, mais qui ont été et continuent d'être fortement influencés par les systèmes de tenure étatique hérités de l'ère coloniale ${ }^{1}$ et post-indépendance. Ce document de travail cherche à combler certaines des lacunes existantes dans les connaissances sur les systèmes coutumiers vivants par le biais d'un examen des études de terrain menées sur les systèmes de tenure tels qu'ils existent à Madagascar, mais aussi d'un aperçu des textes juridiques pertinents. Cet examen permettra d'orienter les efforts en cours pour intégrer les terres détenues collectivement dans la politique et la législation foncières malgaches.

Ce document est divisé en deux parties. La première partie donne un aperçu des textes juridiques susceptibles d'influencer les possibilités de renforcement des systèmes de tenure communautaire. Les textes examinés couvrent :

1. les collectivités territoriales décentralisées (CTD), en se focalisant sur les communes rurales, qui ont la responsabilité d'administrer le système de certification foncière établi par la loi foncière de 2005 de Madagascar ;

2. les fokontany, le plus bas échelon de l'appareil de l'État ;

3. les fokonolona, les assemblées communautaires liées aux fokontany ;

4. les associations communautaires de gestion forestière.

La seconde partie synthétise les principaux modèles et thèmes liés aux systèmes de tenure coutumière « vivante » examinés dans notre étude.

1 Bien que l'accent soit souvent mis sur les conséquences du système colonial français sur les systèmes de tenure foncière malgaches, il faut garder à l'esprit que les systèmes coloniaux autochtones, particulièrement ceux des royaumes des Merina et des Sakalava, ont également influencé les systèmes de tenure foncière coutumières dans de nombreuses parties de l'île. 


\section{Les textes juridiques relatifs à la tenure foncière communautaire}

Nous commencerons par une brève description des catégories de tenure foncière étatique et des divisions administratives de Madagascar pour permettre aux lectrices et aux lecteurs, qui ne connaîtraient pas l'île, de s'y retrouver.

\subsection{Aperçu des catégories de tenure foncière étatique et des divisions administratives}

Avant 2005, la loi foncière malgache était basée sur une présomption de domanialité de l'État. En résumé, toute terre qui n'avait pas fait l'objet d'un titre de propriété par le biais du système de titres fonciers administré par l'État était considérée comme appartenant à l'État. Cela signifie donc que la plupart des terres situées dans les zones rurales de Madagascar, dont la majorité n'ont jamais été titrées et continuent d'être régies de manière informelle par le droit coutumier, étaient considérées comme propriété de l'État. La loi foncière de 2005 a remplacé la présomption de domanialité de l'État par la présomption de propriété privée en vertu du droit coutumier, pour les terres non titrées qui étaient occupées. Ainsi, en vertu de l'article 2 de la loi foncière de 2005, les terres sont divisées en trois grandes catégories : les terres domaniales (publiques et privées); les terres privées sans titre (connues sous le nom de propriété privée, non titrée ou PPNT); et les terres soumises à un régime juridique de protection spécifique (par exemple, les parcs nationaux, les réserves forestières et les aires protégées). En effet, la loi foncière de 2005 reconnaît les droits fonciers coutumiers pour les terres agricoles, les zones résidentielles et les plantations d'arbres, mais pas pour les forêts ou les pâturages (Jones et al. 2018).

Comme le montre la figure 1, Madagascar dispose d'un système de gouvernance parallèle, composé de quatre échelons de circonscriptions administratives déconcentrées (provinces, districts, arrondissements administratifs et fokontany) et de deux types de collectivités territoriales décentralisées (régions et communes). De plus, chaque fokontany dispose d'un fokonolona (ou assemblée communautaire) composé de toutes les personnes âgées de 18 ans révolus et plus qui vivent au sein du fokontany. Les unités déconcentrées sont organisées hiérarchiquement, tandis que les régions et les communes sont autonomes les unes par rapport aux autres ainsi que par rapport aux circonscriptions administratives déconcentrées.

Les 23 régions du pays sont divisées géographiquement en districts. Les districts sont subdivisés en arrondissements administratifs, et chaque district compte plusieurs communes autonomes. Les arrondissements administratifs sont subdivisés en fokontany ; chaque commune compte également plusieurs fokontany. Le chef de région et le maire de la commune, ainsi que les membres du conseil régional et municipal sont élus au suffrage universel. Tant les régions que les communes disposent d'une autonomie financière.

Les fokontany constituent les circonscriptions administratives déconcentrées de l'appareil étatique les plus proches de la majorité de la population rurale malgache. Le chef du fokontany et son adjoint sont choisis par le chef de district sur une liste de candidats fournie par le fokonolona, l'assemblée communautaire associée à chaque fokontany. Le fokonolona correspond très étroitement aux institutions malgaches qui gouvernaient traditionnellement au niveau du village. Cependant, comme nous le décrirons plus en détail dans cette section, on ne sait pas si la législation malgache permet la reconnaissance des fokontany ou des fokonolona comme propriétaires fonciers. 


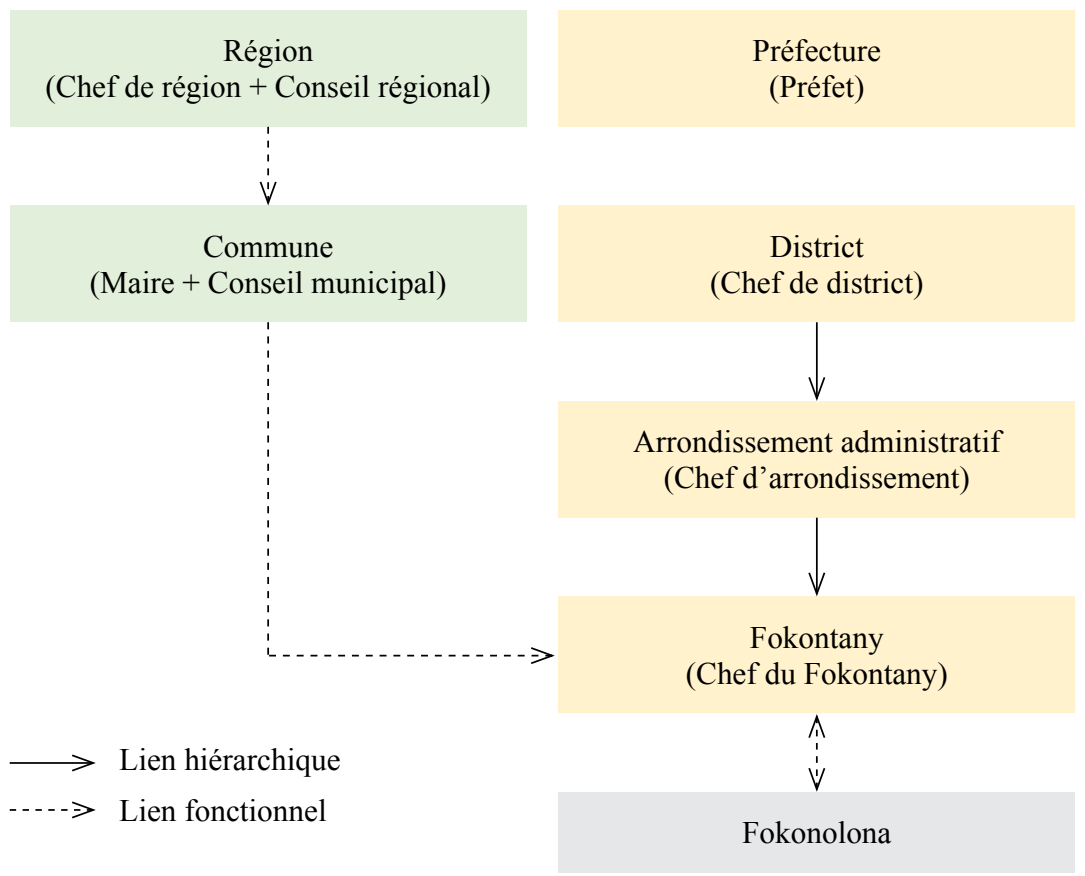

Figure 1. La structure de gouvernance parallèle à Madagascar

Les rectangles verts représentent les collectivités territoriales décentralisées. Les rectangles jaunes représentent les circonscriptions administratives déconcentrées de l'État. Le rectangle gris représente le fokonolona (ou assemblée communautaire), qui est une entité ne relevant pas des pouvoirs publics.

\subsection{Les collectivités au niveau local}

Quatre grands types de collectivités locales (communes, fokontany, fokonolona et communautés de base ou COBA) occupent actuellement un rôle important dans l'administration foncière au sein du cadre juridique étatique. Cette section donne un aperçu de ces collectivités et de leurs rôles dans l'administration foncière, tels qu'ils sont définis et décrits dans les différentes lois, ordonnances et décrets du pays.

\subsection{Les collectivités territoriales décentralisées}

D'après l'article $1^{\text {er }}$ de la loi n ${ }^{\circ}$ 94-008, la collectivité territoriale décentralisée (CTD) est « une portion du territoire national (...) dotée de la personnalité morale et de l'autonomie financière ». En outre, l'article 9.1 de la loi n ${ }^{\circ} 2014-018$ évoque une « solidarité sociologique de chaque Collectivité Territoriale Décentralisée ayant une assise géographique qui implique la prise en considération des facteurs socioculturels ». La loi $n^{\circ}$ 2014-18 du 12 septembre 2014 régissant les modalités d'organisation et de fonctionnement des CTD prévoit trois CTD autonomes (province, région, commune), mais actuellement seules celles des régions et des communes sont fonctionnelles. Nous nous concentrerons ici sur la commune ${ }^{2}$, car c'est la CTD la plus proche des communautés locales.

2 À partir des années 1990, Madagascar a transféré les autorités administratives et financières aux collectivités territoriales décentralisées (CTD). Les CTD de inférieur, les communes, ont été créées en 1996 (Banque mondiale 2004). Les régions, qui comprennent plusieurs communes, ont été créées en 2004 (Bidou et al. 2008). Les communes sont devenues les principales entités de gouvernance décentralisée au niveau local (Bidou et al. 2008). L'article 139 de la Constitution de 2010 confère aux CTD une personnalité morale ainsi qu'une autonomie administrative et financière. L'article 140 confère au CTD un pouvoir réglementaire sur son territoire et le droit à l'autogestion. Le maire, l'agent exécutif de la commune, et les membres du conseil municipal sont élus (Bidou et al. 2008). 


\title{
2.3.1 Le rôle des collectivités territoriales décentralisées dans le système de tenure foncière étatique
}

\author{
Les propriétés privées détenues par des collectivités territoriales décentralisées
}

Les CTD peuvent posséder et gérer des terres enregistrées à leur nom. Ces terres leur permettent d'accomplir leurs fonctions. Dans le cas des communes, ces fonctions comprennent la fourniture de services administratifs, sociaux et sanitaires de base, ainsi que l'administration du système de certification foncière introduit par la loi foncière de 2005 (loi n ${ }^{\circ} 2005-019$ du 17 octobre 2005 fixant les principes régissant les statuts des terres).

\section{L'enregistrement foncier pour les collectivités territoriales}

En vertu de l'article 153 de l'ordonnance $n^{\circ} 60-146$ et de l'article 31 de la loi ${ }^{\circ}$ 60-004, une collectivité territoriale peut demander l'immatriculation collective des terres situées sur son territoire. L'immatriculation collective a débuté sous le régime colonial français par la mise en place d'un cadastre autochtone, une procédure destinée à reconnaître les droits fonciers des Malgaches. En dépit de son appellation, l'immatriculation collective ne vise pas à enregistrer des droits collectifs, mais constitue plutôt une procédure mise en œuvre collective destinée à établir et enregistrer les droits des individus sur les parcelles appartenant aux membres de la communauté concernée. Après l'abrogation de la loi sur le cadastre autochtone à la suite de l'indépendance, les titres cadastraux autochtones ont été transformés en titres fonciers d'État, et toute procédure cadastrale ultérieure s'est poursuivie par le biais de l'immatriculation foncière collective. Bien que la loi précise que la collectivité territoriale initie la procédure d'immatriculation foncière collective, les bénéficiaires finaux des titres de propriété sont les individus qui apportent la preuve de leur occupation ou de l'aménagement des terres. En d'autres termes, dans le cadre de cette procédure, l'immatriculation foncière est mise en œuvre de manière collective, mais les parcelles enregistrées au cours de la procédure sont enregistrées de façon individuelle.

\section{Les concessions de terres de l'État aux collectivités territoriales}

D'après la loi n 60-004 du 15 février 1960, une collectivité territoriale peut demander une concession de terre (donation) appartenant au domaine privé de l'État. La donation est une mise à disposition conditionnelle de terrains assortie de conditions d'aménagement. Ces demandes sont destinées à être utilisées pour des portions du domaine privé de l'État où les résidents de la collectivité territoriale concernée disposent d'un droit de jouissance collective. La procédure est engagée par une commission créée par décret. La donation est également prononcée par décret.

Pendant la procédure de donation, la collectivité a la responsabilité de :

- Résoudre les litiges internes portant sur des parcelles (article 155 de l'ordonnance $n^{\circ} 60-146$ du 3 octobre 1960 relative au régime foncier de l'immatriculation).

- Détermine, par l'intermédiaire du conseil de la collectivité, « les terrains dont l'usage doit rester collectif et ceux qu'il entend réserver en vue d'une mise en valeur collective (...)» (article 37) ; les droits d'usage collectif ne peuvent donner lieu à l'immatriculation du terrain au nom des bénéficiaires (article 54), tandis que les zones d'aménagement collectif peuvent être immatriculées au nom des bénéficiaires.

- Définir la vocation des terres, qui peut être « forestière et protection », « pastorale » ou « agricole et élevage intensif » (article 1er de l'ordonnance $\mathrm{n}^{\circ}$ 62-123).

Les terres obtenues par donation peuvent devenir des propriétés individuelles ou être destinées à un usage collectif, sous réserve de respecter les conditions d'utilisation associées. La collectivité territoriale est la gardienne de l'acte écrit (procès-verbal ou PV) qui confirme l'occupation (c'est-àdire la sécurité foncière collective) et du plan d'occupation collective des terres, et est responsable des modifications futures, comme l'extension des parcelles, etc. 


\section{La gestion et la conservation des terres}

En 1996, la loi n 96-025 a introduit la Gestion Locale Sécurisée (dite loi GELOSE), une forme de cogestion entre les communautés locales et l'État malgache sur les terres appartenant au domaine privé de l'État. Le décret n ${ }^{\circ}$ 98-610 du 13 août 1998 réglementant les modalités de la mise en œuvre de la Sécurisation Foncière Relative (SFR) ${ }^{3}$ précise la procédure de délimitation de l'ensemble du territoire appartenant à une communauté de base bénéficiant d'un accord de cogestion forestière. D'après l'article 10 , dans les communautés où la SFR a été mise en œuvre, la commune conserve les minutes des documents SFR (notamment le plan du périmètre de la zone soumise à la SFR) et l'utilise pour autoriser les nouvelles extensions de parcelles (article 12). En outre, d'après les articles 13 et 14, la commune est l'instance d'arbitrage de second niveau pour les litiges relatifs à la SFR en cas d'échec de l'action de conciliation menée par les communautés de base chargées de la gestion de la zone soumise à la SFR.

En 2001, le décret n ${ }^{\circ}$ 2001-122 relatif à la gestion contractualisée des forêts pour les forêts domaniales a été établi, afin de simplifier le processus d'obtention des contrats de gestion forestière (CGF) par les communautés locales. En vertu de ce décret, la commune n'est plus tenue de signer les contrats de gestion forestière, et les médiateurs environnementaux ne sont plus tenus d'intervenir. Les dinas sont toujours identifiés comme faisant partie intégrante des contrats. Cependant, le décret n'identifie pas la sécurité foncière comme étant nécessaire à la gestion durable et ne mentionne pas l'utilisation de la SFR comme moyen pour la communauté d'obtenir la propriété légale de la terre.

\subsection{Le fokontany}

\subsubsection{Définition et description du fokontany}

Le principal décret fixant la définition et les attributions du fokontany est le décret n ${ }^{\circ} 2004-299$ du 3 mars 2004, modifié en 2007. Le fokontany est la subdivision administrative de base au niveau de la commune : une commune compte ainsi plusieurs fokontany sur son territoire. Les fokontany sont également la subdivision administrative de base des arrondissements administratifs (voir la loi ${ }^{\circ} 2014-021$ relative à la représentation de l'État). Un fokontany peut être un ensemble de hameaux, de villages, de secteurs ou de quartiers. La création d'un fokontany a lieu par décision du représentant de l'État territorialement compétent, à partir d'une liste proposée par le maire après délibération du conseil municipal. Le fokontany est dirigé par un comité composé d'un chef de fokontany et de son adjoint. L'article $5 \mathrm{du}$ décret $n^{\circ}$ 2004-299 précisait que le chef de fokontany était choisi par le maire de la commune sur une liste de cinq noms fournie par les membres du fokonolona. Cependant, le décret $\mathrm{n}^{\circ} 2007-151 \mathrm{du}$ 19 février 2007 a modifié le décret n 2004-299 pour que la sélection du chef de fokontany et de son adjoint incombe au chef de district. Avec ce changement, le fokontany est devenu la circonscription administrative déconcentrée du gouvernement national la plus proche des populations locales.

Chaque fokontany dispose d'une assemblée générale pour prendre des décisions (décret n ${ }^{\circ}$ 2004-299 du 3 mars 2004). L'assemblée générale du fokontany est composée de l'ensemble des habitants du fokontany âgés de 18 ans révolus et plus. Les décisions sont prises à la majorité absolue et nécessitent la présence d'au moins la moitié des membres du fokontany âgés de plus de 18 ans. Le quorum est réduit à un cinquième des membres de plus de 18 ans si moins de la moitié des membres du fokontany ne se sont pas rendus aux deux premières convocations successives à l'assemblée générale.

3 La Sécurisation Foncière Relative (SFR) s'inspirait du Plan Foncier Rural (PFR) de la Côte d'Ivoire, par lequel les communautés devaient cartographier leurs frontières, puis utiliser des photographies aériennes pour cartographier les parcelles individuelles et recenser leurs propriétaires par le biais d'une procédure publique d'attribution des revendications foncières. Les communautés qui ont suivi ce processus jusqu'à la phase officielle d'attribution des titres deviendraient alors les propriétaires des terres cartographiées. Toutefois, peu de communautés ont été en mesure de mener à bien ce processus, qui s'est avéré coûteux et chronophage (Banque mondiale 2006). 
Le chef du fokontany (ou son adjoint en cas d'absence) est chargé de :

- l'administration générale du fokontany ;

- le recouvrement et le reversement des impôts, droits et taxes fiscales à la commune ;

- la convocation (par écrit) de l'assemblée générale du fokontany ;

- la rédaction puis la transmission du procès-verbal de l'assemblée générale à la commune.

\subsubsection{Les rôles du fokontany dans la tenure foncière}

Les rôles du fokontany en matière de tenure foncière sont :

- Le recouvrement des taxes foncières, par extension de son rôle de recouvrement des impôts ;

- La participation au comité de reconnaissance locale chargé de valider les revendications sur les terrains privés non titrés. À cet effet, le chef du fokontany établit annuellement une liste de « raiamandreny » (anciens) proposés par les habitants de celui-ci. Les représentants des fokontany au comité de reconnaissance locale sont choisis sur cette liste (article 11 de la loi $\mathrm{n}^{\circ}$ 2006-031).

\subsection{Le fokonolona}

\subsubsection{Définition et description du fokonolona}

Selon l'article 152 de la Constitution de 2010, « Le Fokonolona, organisé en fokontany au sein des communes, est la base du développement et de la cohésion socio-culturelle et environnementale ». D'autres lois, plus anciennes mais toujours en vigueur, définissent différemment le fokonolona. Ainsi, la loi n 2004-299 du 3 mars 2004, dans son article 2, décrit le fokonolona comme une subdivision $\mathrm{du}$ fokontany, telle qu'un ou plusieurs hameaux ou un ou plusieurs villages. La loi n ${ }^{\circ}$ 2001-004 du 25 octobre 2001 portant réglementation générale des Dina (ou conventions collectives écrites) donne une définition plus nuancée du fokonolona, le décrivant comme une « communauté humaine, spatiale et entité administrative ». L'article 1.2 de cette même loi confirme que le fokonolona correspond aux hameaux, villages et fokontany.

Le décret $\mathrm{n}^{\circ}$ 2002-1170 du 7 octobre 2002 précise que le fokonolona est une cellule de gestion administrative locale (article 2), composée de « petites agglomérations urbaines ou districts ruraux, en l'occurrence des hameaux, villages ou îlots défendant les mêmes intérêts, et ayant l'habitude d'unir leurs efforts pour atteindre des objectifs communs ». Par ailleurs, le fokonolona dispose d'un président et un vice-président (article 20) élus au suffrage universel par les membres de plus de 18 ans révolus. Le comité du fokontany veille à l'élection du comité du fokonolona (article 21).

Le fokonolona choisit les membres du comité du fokontany (par exemple, le chef du fokontany et son adjoint) sur une liste de cinq personnes proposées. Ce choix se fait lors d'une assemblée générale du fokonolona convoquée par le maire de la commune (article 5 du décret n ${ }^{\circ}$ 2004-299 du 3 mars 2004). Selon la loi n ${ }^{\circ} 2001-004$ du 25 octobre 2001, le fokonolona peut créer un dina en assemblée générale, à l'échelle d'un hameau, d'un village ou d'un fokontany. Mais, d'après l'article 8 de cette loi, le dina qui voit le jour doit être approuvé respectivement par le conseil municipal ou communal, le représentant de l'État et le tribunal de l'ordre judiciaire territorialement compétent.

\subsubsection{Les rôles du fokonolona dans la tenure foncière}

Dans de nombreuses procédures foncières, le fokonolona ou ses représentants sont appelés à participer aux visites sur le terrain pour valider les revendications de propriété. Selon l'article 89 de l'ordonnance $\mathrm{n}^{\circ}$ 60-146 du 3 octobre 1960, un représentant du fokonolona est invité par les services fonciers de l'État, par l'intermédiaire du chef de district, à assister au processus de bornage des parcelles. Cependant, ni le fokonolona ni son représentant ne sont mentionnés à l'article 90 comme faisant partie de la liste des personnes assistant au bornage. Le fokonolona est également convoqué pour assister aux réunions de la commission de reconnaissance des droits de propriété (article 22 de la 
loi 60-004 du 15 février 1960). Le fokonolona dûment convoqué assiste également la commission lors de la vérification sur place de la procédure d'attribution des concessions (article 48 de la loi $\mathrm{n}^{\circ}$ 60-004 du 15 février 1960 relative au domaine privé national). Lors de la procédure de détermination de la vocation des terres rurales, qui est une étape de la procédure de demande de donation, le fokonolona n'est consulté que si la commission en charge de la procédure le juge nécessaire (décret $n^{\circ} 72-272 \mathrm{du}$ 21 juillet 1972).

Selon l'article 41 de la loi n ${ }^{\circ}$ 97-017 du 8 août 1997 portant révision de la législation forestière, des droits limités sur l'utilisation des forêts sont accordés aux fokonolona. Cet article dispose ainsi que « les membres du Fokonolona sont autorisés à exercer leurs droits d'usage traditionnels individuellement ou collectivement dans les forêts de l'État, des collectivités territoriales décentralisées, des établissements publics et des personnes privées dans la mesure où lesdits droits n'auront pas déjà été purgés ».

\subsection{Les groupes légalement constitués : Vondron'olona ifotony (communautés de base en malgache)}

Cette section se concentre sur les associations de gestion forestière, qui sont autorisées à conclure des contrats de cogestion avec le service forestier malgache ou des entités (telles que les organisations de conservation) auxquelles ce service a délégué la responsabilité de la gestion.

\subsubsection{Définition et description des groupements légalement constitués}

D'après l'article 6 de la loi n 2006-031 du 24 novembre 2006, « un groupement d'occupants constitué conformément aux dispositions légales en vigueur », comme une association, peut demander collectivement la reconnaissance des droits de propriété privée sur les terrains non titrés occupés. D'après l'article 8 , les droits peuvent être reconnus pour le groupement lui-même ou pour chacun de ses membres. L'article 9 de la même loi précise que la demande peut émaner d'un représentant légal du groupement ou d'une personne déléguée à cette fin dans les conditions prévues par les statuts du groupement. Un cas particulier concerne « la communauté de base» (COBA) ou « Vondron'olona ifotony » (VOI) en malgache, créée pour mettre en œuvre la loi GELOSE. C'est cette loi qui régit le transfert des droits de gestion des ressources naturelles renouvelables (voir la note de bas de page 1 pour une description de la loi GELOSE).

\subsubsection{Le contrôle des ressources}

La VOI exerce des droits d'usage définis dans le Plan d'aménagement et de gestion (PAG), et conformément aux règles qu'il établit pour des zones d'utilisation spécifiques. Ces droits d'usage sont normalement autorisés pour tous les membres de la communauté, mais ils peuvent être soumis à des taxes imposées par la VOI. Les droits d'usage sont des droits collectifs, puisque la ressource transférée n'est pas sujette à une appropriation individuelle par quiconque, qu'il soit membre du VOI ou non. La VOI fonctionne comme un responsable de la ressource dont elle a la responsabilité de gérer. Pour lui permettre d'exercer ses responsabilités, la VOI doit établir un dina qui fixe les règles d'utilisation des ressources, et un organe de surveillance pour surveiller l'application du dina. La VOI organise et effectue des patrouilles de surveillance et alerte les pouvoirs publics en cas d'infraction qui ne relève pas de sa compétence.

\subsubsection{Les outils de gestion des ressources}

Les outils de gestion qui accompagnent le contrat de transfert de gestion sont le plan d'aménagement et de gestion simplifié (PAG) et le dina. L'utilisation de ces outils correspond à l'exercice d'un droit de gestion, tel que conceptualisé dans le modèle du faisceau de droits de Schlager et Ostrom (1992). Les droits de gestion sont déterminés par des négociations entre les services techniques compétents et la VOI, dans lesquelles ils cherchent à concilier les normes techniques de durabilité avec les besoins 
des communautés locales. La loi GELOSE prévoit la possibilité pour les parties de faire appel à un médiateur environnemental pour les assister dans les négociations. Le médiateur est une personne formée pour assurer un dialogue constructif entre les deux parties pendant l'élaboration du contrat de transfert de gestion.

\subsection{La communauté et la tenure foncière}

\subsubsection{Les facteurs limitant les droits fonciers des communautés locales}

Les collectivités territoriales et autres coopératives, comme les associations et les organisations non gouvernementales (ONG), disposent d'un statut juridique, donc d'une personnalité juridique, et peuvent ainsi revendiquer des droits sur les ressources et les terres. Il n'y a rien de surprenant ni d'illogique à cela. Cependant, les collectivités locales informelles de la société civile ne bénéficient pas des mêmes avantages. En effet, les textes juridiques restent vagues lorsqu'il s'agit de savoir si les fokonolona disposent d'une personnalité juridique. Dans les textes, les définitions du fokonolona sont incohérentes et parfois même contradictoires. Pourtant, vu le nombre de lois qui s'y réfèrent, il est clair que les auteurs des textes juridiques considèrent le fokonolona comme une entité sociale importante.

Dans son préambule, la loi $\mathrm{n}^{\circ}$ 2001-004 expose que l'on ne peut ignorer ou se passer du dina, un argument qui plaide indirectement en faveur du fokonolona, puisque le dina était traditionnellement son outil. Des contradictions apparaissent cependant dans la définition du fokonolona ou de ses attributions. La Constitution de 2010 considère que le fokonolona est constitué de l'ensemble des habitants du fokontany, ce qui est aussi explicitement indiqué à l'article 2 du décret n ${ }^{\circ}$ 2004-299. Cependant, d'autres textes donnent une définition moins rigide, et varient en fonction de la division territoriale ou administrative. Mais, même ces définitions plus souples, bien que plus proches de la réalité du terrain, restent insuffisantes, car elles réduisent ou confondent le fokonolona avec les collectivités locales ou les districts administratifs.

Une autre interprétation possible de l'objectif du fokontany est qu'il organise le fokonolona afin que ce dernier se conforme à l'administration de l'État (que le fokontany représente), ainsi qu'aux politiques et aux lois du pays. De ce point de vue, le fokonolona peut être considéré comme une simple entité administrative ${ }^{4}$. Compte tenu de la confusion des rôles et des responsabilités du fokontany et du fokonolona, le fokonolona ne peut logiquement pas posséder ou gérer une terre ou une ressource, à moins que cette terre ne relève du domaine de l'État.

En ce qui concerne les attributions du fokonolona, il y a quelques points importants à noter, comme la possibilité pour « les habitants du fokonolona » de souscrire une assurance collective (décret $\mathrm{n}^{\circ} 2002$ 1170). On pourrait penser que cela est plausible, puisque la composition du fokonolona est délimitée et qu'il dispose d'un comité (décret $n^{\circ}$ 2002-1170). Mais si tel est le cas, la question de la personnalité juridique subsiste : comment une entité sans existence juridique peut-elle être agréée par un assureur ? Bien que cette interprétation puisse être remise en question, elle se fonde sur ce qui est énoncé dans les textes juridiques, et ces derniers ne peuvent se permettre de donner lieu à de telles interprétations erronées. En d'autres termes, tant que le flou et les contradictions potentielles persisteront autour du concept de fokonolona, ce dernier ne pourra pas exercer les droits d'attribution et de gouvernance des terres, car il est juridiquement déconseillé, et même impossible, d'attribuer des droits à une entité qui n'est pas clairement définie.

4 Le fokonolona sélectionne cinq membres qui se présenteront à l'élection du fokontany, tandis que le comité du fokontany veille à l'élection du président du fokonolona. 


\subsubsection{Les droits individuels face à la gouvernance collective}

Les textes juridiques, que ce soit sur la tenure foncière ou les secteurs connexes, sont suffisamment explicites : le législateur malgache privilégie clairement le renforcement des droits individuels sur les droits collectifs. De même, des expressions telles que « immatriculation collective », « aménagement collectif », « droit de jouissance collective », qui de prime abord semblent soutenir l'immatriculation collective et abondent dans les textes, sont trompeuses. En effet, elles donnent l'impression erronée qu'elles se rapportent à des utilisations véritablement collectives, soumises à une gouvernance collective. Or, dans la plupart des cas, elles font référence aux droits des individus sur des parcelles individuelles.

L'utilisation de ces expressions trompeuses porte préjudice au concept de droits d'usage collectif, un terme qui n'apparait que rarement dans les textes. En matière de sécurisation des droits, les textes privilégient l'enregistrement, qu'ils considèrent comme l'idéal. Une affirmation telle que « les droits d'usage ne peuvent donner lieu à immatriculation directe au nom des bénéficiaires » (article 54 de la loi $\left.\mathrm{n}^{\circ} 60-004\right)$ laisse penser que les rédacteurs de la loi ont éprouvé un certain dédain à l'égard de ces usages collectifs qui existent pourtant. De plus, le soutien à l'organisation et à la gouvernance locales spontanées et traditionnelles concernant ces usages dits collectifs est totalement passé sous silence dans les textes de loi. Même la loi GELOSE, qui est actuellement la seule loi à reconnaître le droit des communautés locales à gérer les ressources, s'appuie sur le modèle de la communauté de base dotée de la personnalité juridique, un concept qui ne doit pas être confondu avec une véritable communauté locale.

\subsection{Les textes juridiques cités}

Constitution de la IVe République du Madagascar. 11 decembre 2010

Loi $\mathrm{n}^{\circ}$ 60-004 du 15 février 1960 relatif au domaine privé national

Loi $\mathrm{n}^{\circ}$ 93-005 du 26 janvier 1994 portant orientation de la politique de décentralisation, modifiée par la Loi $\mathrm{n}^{\circ}$ 94-039 du 3 Janvier 1995, et la Loi no 2004-001 du 17 Juin 2004

Loi $\mathrm{n}^{\circ}$ 94-008 du 26 avril 1995 fixant les règles relatives à l'organisation, au fonctionnement et aux attributions des CTD [collectivités territoriales décentralisées]

Loi $\mathrm{n}^{\circ}$ 96-025 du 30 septembre 1996 relative à la gestion locale des ressources naturelles renouvelables

Loi $n^{\circ}$ 97-017 du 8 août 1997 portant révision de la législation forestière

Loi n ${ }^{\circ}$ 2001-004 du 25 octobre 2001 portant réglementation générale des Dina en matière de sécurité publique

Loi $\mathrm{n}^{\circ} 2004-001 \mathrm{du} 17$ juin 2004 relative aux Régions

Loi $\mathrm{n}^{\circ} 2005-019$ du 17 octobre 2005 fixant les principes régissant les statuts des terres

Loi $\mathrm{n}^{\circ}$ 2006-031 du 24 novembre 2006 fixant le régime juridique de la propriété foncière privée non titrée

Loi n ${ }^{\circ} 2014-021$ du 12 septembre 2014 relative à la représentation de l'Etat

Loi organique $\mathrm{n}^{\circ}$ 2014-018 du 12 septembre 2014 régissant les compétences, les modalités d'organisation et de fonctionnement des Collectivités territoriales décentralisées, ainsi que celles de la gestion de leurs propres affaires

Décret $\mathrm{n}^{\mathrm{o}} 72-272 \mathrm{du} 21$ juillet 1972 portant sur le classement en zones à vocations forestières, pastorales ou agricoles des terres de Madagascar

Décret $n^{\circ}$ 98-610 du 13 août 1998 règlementant les modalités de la mise en œuvre de la Sécurisation Foncière Relative

Décret $n^{\circ}$ 2001-122 du 14 février 2001 fixant les conditions de mise en œuvre de la gestion contractualisée des forêts de l'Etat

Décret no 2002-1170 du 9 octobre 2002 fixant les règles relatives à l'organisation, au fonctionnement et aux attributions du Fokontany et du Fokonolona 


\section{Suite}

Décret no 2004-299 du 3 mars 2004 fixant l'organisation, le fonctionnement et les attributions du Fokontany

Décret $n^{\circ}$ 2007-151 du 19 février 2007 modifiant certaines dispositions du Décret n ${ }^{\circ}$ 2004-299 du 3 mars 2004 fixant l'organisation, le fonctionnement et les attributions du Fokontany

Ordonnance $\mathrm{n}^{\circ} 60-146$ du 3 octobre 1960 relative au régime foncier de l'immatriculation

Ordonnance $n^{\circ} 62-123$ du 1 octobre 1962 sur la vocation des terres rurales

Ordonnance $\mathrm{n}^{\mathrm{o}}$ 73-073 portant orientation du développement rural

Ordonnance $\mathrm{n}^{\circ}$ 74-021 portant refonte de l'ordonnance $\mathrm{n}^{\circ}$ 62- $110 \mathrm{du}$ ler octobre 1962 sanctionnant l'abus de droit de propriété et prononçant le transfert à l'Etat des propriétés non exploitées. 


\section{Les grands axes de la gouvernance foncière au niveau local à Madagascar}

\subsection{Introduction}

Une littérature abondante existe sur la gouvernance et les régimes fonciers coutumiers à Madagascar, dont une grande partie est basée sur des recherches ethnographiques et géographiques réalisées à partir des années 1960 par l'Institut de Recherche pour le Développement (IRD), anciennement Office de la Recherche Scientifique et Technique Outre-mer (ORSTOM) ${ }^{5}$. Pour que la portée de notre étude reste faisable, nous nous sommes principalement concentrés sur les études publiées après 1990 et qui portaient au moins sur les questions de gouvernance et de tenure liées aux terres ou aux ressources gérées collectivement, comme les pâturages et les forêts. Nous avons examiné des études provenant de plusieurs régions afin d'englober un éventail de systèmes de tenure coutumière, de systèmes de production et d'écosystèmes existants à Madagascar. Nous avons aussi plus généralement analysé des études sur les systèmes de tenure, ainsi que celles sur des zones où les villages ont conclu des accords de cogestion concernant les parcs nationaux, les réserves spéciales, les forêts classées, ainsi que les forêts et pâturages classés comme terres appartenant au domaine public. La Figure 2 (à la fin de ce rapport) montre les 22 régions de Madagascar et la Figure 3 indique la localisation d'un échantillon sélectionné d'études. Notre étude n'a pas la prétention d'être une revue exhaustive des recherches sur la gouvernance et la tenure coutumières à Madagascar, mais elle vise à éclairer le lecteur sur les similitudes et les différences des systèmes de gouvernance et de tenure à travers l'île.

\subsection{Le pluralisme juridique : à la croisée des systèmes étatiques et coutumiers en matière de tenure et de gouvernance}

L'organisation de la gouvernance dans les zones rurales de Madagascar est caractérisée par un pluralisme juridique, des systèmes juridiques étatiques et coutumiers fonctionnant simultanément (Boué et Colin 2018). Cependant, le pluralisme juridique ne signifie pas que les systèmes étatiques et coutumiers fonctionnent indépendamment les uns des autres. Le pluralisme juridique à Madagascar consiste plutôt en un système hybride étatique et coutumier (Boué et Colin 2018 ; Valkonen 2018 ; Di Roberto 2020). Les contrats de cogestion forestière autorisés par la loi GELOSE de 1996 sont des exemples de systèmes hybrides, dans lesquels les règles coutumières peuvent réglementer l'accès aux produits forestiers, à condition qu'elles ne contreviennent pas aux lois ou politiques nationales (Henkels 2002).

D'autres exemples sont moins fréquents, comme les ventes de terres qui ne suivent pas les procédures officielles de l'État pour l'enregistrement des transactions foncières, mais qui sont enregistrées par le biais de documents écrits, signés et tamponnés par des fonctionnaires locaux, qui peuvent être le chef du fokontany et/ou le maire de la commune (Di Roberto 2020). À Madagascar, le droit coutumier a traditionnellement été transmis oralement, et continue à l'être dans certaines régions. Cependant, l'établissement de documents écrits pour les transactions foncières (Boué et Colin 2018 ; Di Roberto 2020) et la codification des règlements des communautés locales régissant les ressources forestières sont de plus en plus la norme (Henkels 2002).

5 Pour un aperçu des recherches produites via l'ORSTOM-IRD jusqu'au début des années 2000, consulter la publication de Feller et Sandron (2010). 


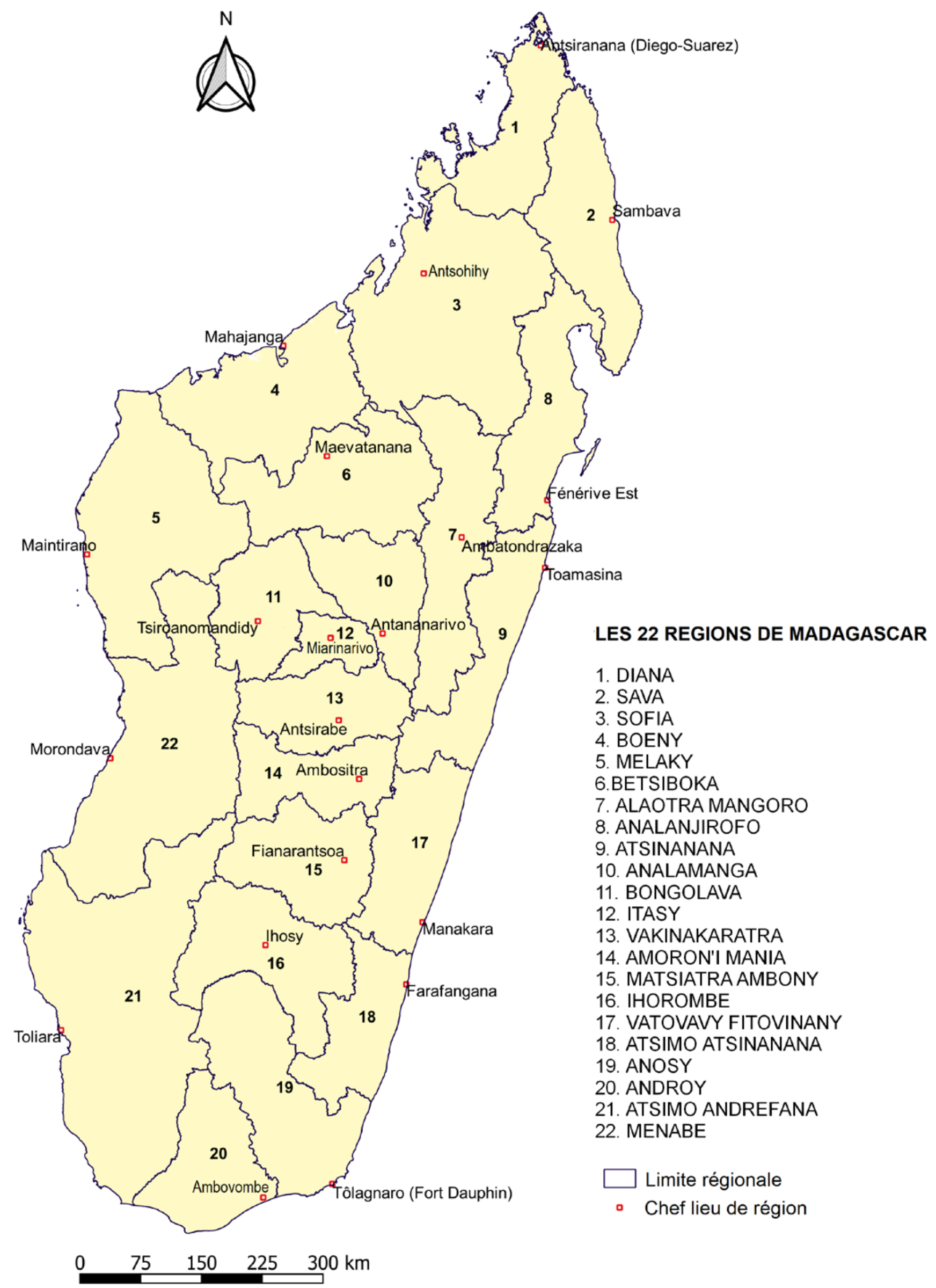

Figure 2. Les régions de Madagascar 

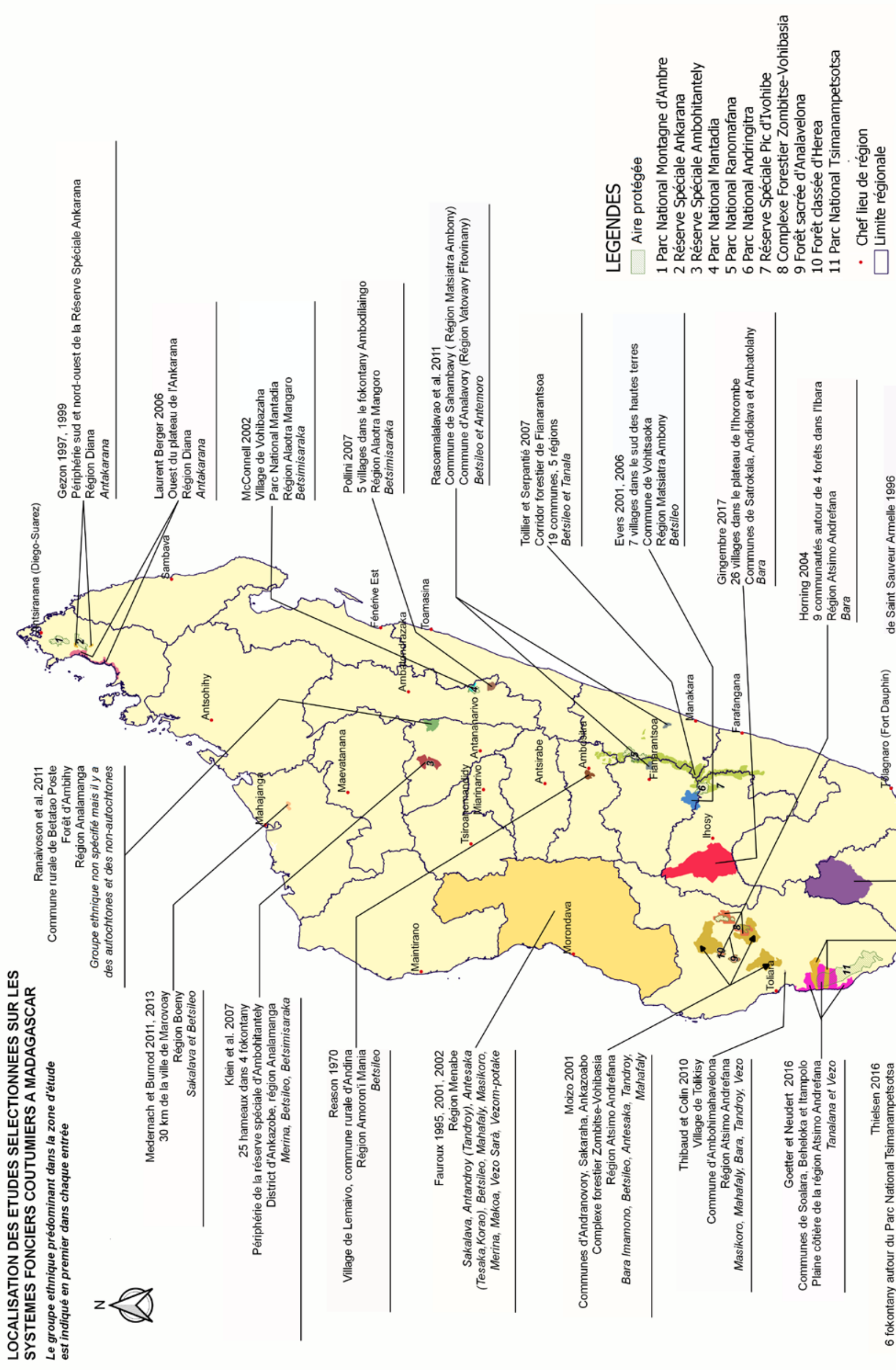
La relation entre les autorités étatiques et traditionnelles dans les zones rurales de Madagascar est complexe et caractérisée par une interdépendance mutuelle. Les autorités traditionnelles peuvent avoir besoin du soutien des agents de l'État, particulièrement lorsqu'elles souhaitent appliquer les lois locales qui restreignent l'utilisation des ressources forestières ou des parcours aux personnes qui ne sont pas originaires du lieu (Horning 2004). Toutefois, ce soutien n'est pas toujours au rendez-vous. Ainsi, Muttenzer (2006) documente un cas dans la région de Boeny, dans le nord-ouest de Madagascar, où les efforts des villageois pour empêcher les personnes venues d'ailleurs de convertir des parties de leur forêt sacrée en champs agricoles ont été contrecarrés par le refus des agents de l'État de faire respecter l'interdiction de défricher la forêt. À l'inverse, les représentants de l'État peuvent avoir besoin du soutien des chefs traditionnels lorsqu'ils délivrent des concessions foncières pour des aménagements agricoles ou forestiers à grande échelle, l'exploitation minière ou forestière. L'étude de Gingembre de 2017 sur les tractations d'une société d'investissement pour acquérir un bail à long terme portant sur des terres situées sur le plateau d'Thorombre illustre comment les autorités de l'État et du village (connues sous le nom de « lonaky ») travaillent ensemble pour promouvoir leurs intérêts. Elle déclare ainsi :

«Si les maires (des communes) peuvent gagner en légitimité en étant proches de chefs locaux respectés, les « lonaky » ont généralement besoin du soutien des agents de l'État pour exercer une influence sur les affaires extérieures. Dans cette relation d'interdépendance, l'équilibre peut varier d'un cas à l'autre» (Gingembre 2017:40).

Lorsque les autorités traditionnelles conservent une légitimité locale, elles fonctionnent souvent comme des intermédiaires entre les membres de la communauté et les autorités extérieures (Evers 2001 ; Horning 2004 ; Gingembre 2017 ; Di Roberto 2020). Cependant, dans ce rôle, les autorités traditionnelles agissent parfois de manière à favoriser leurs propres intérêts politiques et économiques, au lieu de travailler au profit de la communauté au sens large (Blanc-Pamard et Fauroux 2004 ; Berger 2006 ; Gingembre 2017).

Le soutien des autorités traditionnelles peut faciliter les missions d'organisations internationales de conservation, telles que celle du World Wide Fund (WWF) ou de Conservation International, auxquelles l'État a délégué les pouvoirs de gestion en matière de parcs nationaux ou d'aires protégées. Berger (2006) rapporte que le WWF a pu obtenir un certain succès dans la réduction de la déforestation d'une aire protégée sur le plateau de l'Ankarana, dans le nord de Madagascar, en travaillant par l'intermédiaire du roi (« ampanjaka ») des Antankaranana, le groupe ethnique dominant, pour promouvoir le projet. Dans certaines régions, les frontières entre l'État et les systèmes coutumiers sont si floues qu'il est difficile de les différencier. C'est le cas sur le plateau de l'Ankarana et les zones côtières adjacentes, où la monarchie Antankaranana a été réorganisée en une association ayant un statut légal dans les années 1990 (Berger 2006). Le rôle de l'association consiste à protéger et à préserver les espaces sacrés, à enseigner aux jeunes générations les coutumes et la culture Antankaranana, et à protéger l'environnement. L'association est organisée autour de la structure administrative du fokontany et l'adhésion est ouverte aux migrants récents et de longue date, ce qui leur permet de participer à la prise de décisions sur les questions foncières, dont ils étaient auparavant exclus. Berger (2006) affirme que l'association a essentiellement remplacé la monarchie par une instance démocratique centralisée, composée d'agents royaux qui représentent l'État.

\subsection{La gouvernance locale traditionnelle}

Les systèmes de gouvernance locale traditionnelle à Madagascar varient en fonction du groupe ethnique ${ }^{6}$ qui est ou était historiquement dominant dans une région, ainsi que de la capacité du groupe à conserver son autorité traditionnelle face aux pressions exercées par l'État, les flux migratoires, et l'évolution culturelle lié à la transformation structurelle des économies locales (cf. Fauroux 2001, 2002 ; Moizo 2001 ; Thibaud 2010). Comme l'illustre le tableau 1, Madagascar compte 18 catégories ethniques officiellement

6 Dans le nord de Madagascar, le terme « karazaña » est utilisé pour faire référence à l'« ethnie » d'une personne (Brown 2004) mais peut également faire référence au clan d'une personne (Lambek et Walsh 1997). 
Tableau 1. Les groupes ethniques malgaches

\begin{tabular}{lll}
\hline Antaifasy & Antanosy & Makoa (Masombika) \\
Antaimoro & Bara & Merina \\
Antaisaka & Benzanozano & Sakalava \\
Antakarana & Betsileo & Sihanaka \\
Antambahoaka & Betsimisaraka & Tanala \\
Antandroy (Tandroy) & Mahafaly & Tsimihety \\
\hline
\end{tabular}

reconnues (Covell 1987, cité dans Sharp 1993), chacune ayant sa propre gouvernance foncière et son propre système de tenure ${ }^{7}$. Certaines sources traitent les Mikea et les Vezo comme des groupes ethniques distincts tandis que d'autres les considèrent comme des sous-groupes des Sakalava (Sharp 1993).

En matière de tenure foncière, l'appartenance ethnique est importante, car elle peut servir de justification pour autoriser certaines personnes à accéder aux terres et aux ressources ou bien les exclure. L'appartenance à un lignage et à un clan est généralement déterminante dans l'attribution, l'accès et les conditions d'accès aux terres, ainsi que dans la prise de décisions concernant la terre et l'application des règles relatives à l'utilisation des terres et des ressources.

\subsubsection{Le clan : autorité culturelle et religieuse}

Les clans ${ }^{8}$ sont composés d'individus ou de familles qui revendiquent un ancêtre commun, réel ou symbolique (Barry et al. 2000). Le clan fondateur d'un territoire est composé des familles qui se sont initialement installées dans la région. Les clans partagent un nom ou un symbole, des « fady 》 (tabous, appelés « faly » dans certaines régions) et des traditions orales. Entraide, protection mutuelle et hospitalité sont attendues de la part des membres du clan. Le chef du clan, généralement un homme âgé, est une autorité religieuse et sert d'intermédiaire entre les humains et les esprits. Son rôle peut consister à organiser des cérémonies, à résoudre des conflits et à réprimander les comportements sociaux incorrects. Dans les zones de forêts sacrées, le chef de clan est chargé de les gérer; il peut également jouer un rôle important en influençant les comportements locaux à l'égard des forêts protégées (voir Gezon 1997, 1999 ; Berger 2006).

\subsubsection{Le lignage : organisation socio-politique et gouvernance}

Un lignage est constitué des descendants d'un parent commun, chaque membre pouvant retracer son ascendance jusqu'à ce parent (Barry et al. 2000). Dans la plupart des régions rurales de Madagascar, le lignage constitue l'unité de base de l'organisation sociale et politique. Généralement, le chef de lignage, habituellement l'homme le plus âgé, fixe les règles d'utilisation des terres détenues au niveau du lignage. Si un lignage se subdivise, les responsabilités de gouvernance sont gérées au niveau du sous-lignage. Les autorités politiques du village sont souvent constituées des chefs de lignage présents dans le village. Dans la plupart des régions, les chefs de lignage sont appelés « rey amin'dreny », ce qui signifie littéralement « parents $»^{9}$, mais qui est mieux traduit par « anciens du village ». Dans

7 Southall (1971) a remarqué que la plupart des noms donnés aux différents groupes ethniques malgaches l'ont été par des étrangers, et beaucoup de ces noms correspondent à des descriptions environnementales des zones où les différents groupes étaient dominants au moment de la colonisation. Par exemple, «Tanala » = peuple de la forêt ; "Androy » = peuple des ronces ; «Tanosy » = peuple de l'île ; «Ankarana » = peuple des rochers ; et « Sakalava »= peuple de la longue vallée. Dans la mesure où tous ces groupes parlent une variante dialectale malgache, Southall soutient qu'il vaudrait mieux les considérer comme des groupements régionaux ayant des approches adaptatives particulières aux écosystèmes dans lesquels ils résident, plutôt que comme des groupes ethniques. Une récente étude linguistique menée par Serva et Pasquini (2020) abonde dans le sens de son argument concernant les groupements régionaux plutôt que les groupes ethniques.

8 Les informations relatives aux clans et aux lignages figurant dans les sections suivantes sont extraites de plusieurs sources examinées pour ce rapport. Il existe quelques différences, selon le groupe ethnique et le contexte socio-écologique, mais le modèle général des clans et des lignages s'applique à toute l'île.

9 À Ibara, les anciens du village sont appelés « olobe » ou « mpitakazomanga » (Horning 2004). 
certaines communautés, seuls les chefs de familles ou lignées fondatrices sont considérés comme des « rey amin'dreny ». Les familles fondatrices sont généralement appelées « tompon-tany », ou « maîtres de la terre $»^{10}$. Les « tompon-tany », dirigés par leurs membres les plus âgés, ont une compétence générale sur les terres qu'eux-mêmes et leurs ancêtres ont défrichées, ainsi que sur toutes les terres restant à mettre en valeur. L'encadré 1 fournit des exemples de la variété de cette structure de gouvernance foncière généralisée à Madagascar.

De nombreuses études (cf. Ottino 1998 ; Evers 2006 ; Di Roberto 2020) soulignent l'importance de la localisation des tombeaux familiaux pour les sociétés malgaches, cette localisation établissant des droits sur la terre en lui donnant le statut de terre ancestrale ( « tanindrazana »). Dans son étude sur les liens entre parenté, alliances et patrimoine, Ottino (1998) a constaté que, dans tout le pays, la parenté ne peut être dissociée des propriétés foncières, le tombeau familial servant de point d'ancrage aux liens de parenté. Cependant, ce n'est pas n'importe quelle terre qui est importante, mais plutôt la terre ancestrale qui a été transmise par héritage et que les propriétaires ont la responsabilité de garder intacte ou d'accroître afin de pouvoir la transmettre à leur tour à leurs héritiers.

Di Roberto (2020) a constaté que chez les Merina, dans les Hautes Terres du Centre, les relations de parenté sont fondées sur des liens communs avec la terre ancestrale plutôt que sur l'appartenance à une lignée commune. Ainsi, pour les personnes qui ne partagent pas d'ancêtre commun, les relations de parenté découlent du mariage ou d'un pacte de sang ( « fatidra »). Di Roberto a identifié quatre niveaux pour comprendre les droits et les responsabilités en matière de tenure chez les Merina à Ambatomena (région de Vanikaranatra) : le foyer («tokantrano »), la « petite famille» («mpianakavy »), les cohéritiers («mpiara mandova ») et le groupe chargé de veiller au respect des obligations sociales (« mpiaray adidy/mpiara mitrondra »). Le foyer est constitué des membres de la famille nucléaire qui résident dans la même maison ; la « petite famille » est constituée de la famille nucléaire et de ses enfants, qu'ils vivent ou non au sein de la même maison. Les cohéritiers sont des personnes qui ont hérité d'une parcelle de terre commune. L'homme le plus âgé dans la ligne de succession possède le plus d'autorité sur les terres détenues en commun, mais il attribue généralement des parcelles spécifiques à chacun des cohéritiers. Le groupe des coresponsables est constitué des personnes qui partagent les mêmes obligations familiales d'honorer les ancêtres et qui ont la responsabilité conjointe de veiller à ce que le patrimoine du groupe reste intact. L'homme le plus âgé des coresponsables veille au maintien de la cohésion sociale ( «fihavanana ») entre les membres de la famille, et doit être consulté avant que les membres de la famille puissent vendre leurs terres.

Les « rey amin'dreny » sont généralement les principaux décideurs du fokonolona, le conseil de village entériné par l'État et légitime localement, qui gouverne au niveau du fokontany. Dans une étude portant sur les voix qui ont compté dans les décisions relatives à l'attribution de terres communautaires à des concessions foncières à grande échelle sur le plateau d'Ihorombe (situé dans les Hautes Terres du Sud), Gingembre (2017) a conclu que les personnes qui participent aux décisions communautaires varient selon l'endroit, et que celles qui décident véritablement représentent rarement l'ensemble de la communauté. Elle a constaté que les hommes plus âgés avaient tendance à contrôler le vote dans le fokonolona. Les décisions étaient souvent prises par les principaux anciens (c'est-à-dire généralement les hommes chefs de lignées ou de familles fondatrices) à huis clos, avant les réunions communautaires. Dans de tels cas, les réunions communautaires devenaient une formalité, où la politique et les règles étaient proclamées plutôt que débattues et votées.

10 Dans sa recherche sur les communautés Antankarana dans le nord de Madagascar, Walsh (2002:457) souligne la notion de responsabilité pour la terre qui est sous-entendue dans le terme « tompon-tany » : «[Ê]tre tompontany signifie agir de manière responsable sur la terre dont on tire sa subsistance. En effet, compte tenu des multiples significations du terme malgache 'tompo', nous pourrions considérer que les 'tompontany' sont des personnes 'responsables de la terre'. Il est important de comprendre, cependant, que pour beaucoup, être responsable et redevable de la terre à Ankarana équivaut à être responsable et redevable de l'autorité royale ». 


\section{Encadré 1. Exemples de structures de gouvernance foncière}

Zone périphérique de la Réserve Spéciale d'Ambohitantely (Klein et al. 2007) : Dans les hameaux qui composent la « zone périphérique » à l'est de la Réserve Spéciale d'Ambohitantely, au nord d'Antananarivo, l'organisation sociale est basée sur le système de castes des premiers colons Merina. Les chefs de cette zone sont à la fois des anciens et des chefs traditionnels. La « tarika », terme traditionnel utilisé pour désigner la famille, comprend toutes les personnes qui ont un ancêtre commun dans la même tombe. Le chef de la tarika jouit d'un grand prestige dans la société et exerce un pouvoir important au niveau local. Les responsabilités du chef de la tarika vont de la résolution des conflits à l'organisation des cérémonies et rituels traditionnels. Les décisions prises par la commune sans la participation du chef de la tarika sont susceptibles de se heurter à la résistance des autres membres de la communauté qui ne les respecteront pas. Le fokonolona est composé des chefs des différentes tarikas. Les tarikas sont organisées en entreprises sur la base des liens traditionnels créés par un rituel de mélange des sangs et des alliances matrimoniales. Les entreprises s'entraident pour les activités nécessitant une main-d'œuvre fiable, comme la garde du bétail et la plantation du riz.

Vohibazaha (McConnell 2002) : Dans la communauté Betsimisaraka de Vohibazaha, près du parc national de Mantadia, la gouvernance foncière est organisée autour des tombeaux familiaux. La terre est gérée en cercles concentriques, dont la gestion est de moins en moins stricte à mesure que l'on s'éloigne des tombeaux familiaux. La gestion des terres est dirigée par les chefs de lignage/clan (« tangalamena »), qui veillent également au respect des règles sacrées. La communauté Vohibazaha comprend de nombreux lignages. Les chefs de lignage décident à la fois de la politique et de la gouvernance foncière, en étant aussi des chefs religieux et culturels, brouillant ainsi la distinction entre l'autorité du lignage et celle du clan, et entre les familles/lignages nucléaires et étendus. Cette communauté est dirigée par un groupe de 12 chefs de lignage qui gèrent ensemble trois zones principales concentrées autour de sept tombeaux.

Le parc national de Tsimanampetsotsa (Thielsen 2016) : Dans le fokontany situé à l'extrémité nord du parc national de Tsimanampetsotsa, dans le sud-ouest de Madagascar, les Tanalana locaux utilisent le terme fokonolo plutôt que fokonolona. Le fokonolo peut être considéré comme « l'ensemble des personnes qui se réunissent à un moment donné pour prendre une décision ». Il peut prendre deux formes :

1. Fokonolona avec une identité clanique/lignagère : c'est le cas lorsque le fokonolo est convoqué par le chef traditionnel ou les anciens, et comprend les représentants d'un lignage ou d'un clan, qui peuvent vivre dans un ou plusieurs fokontany; ou

2. Fokonolo du fokontany : c'est le cas lorsque le fokonolo est convoqué par le chef du fokontany et comprend les représentants du territoire administratif du fokontany. Le fokonolo convoqué par le fokontany peut comprendre plusieurs groupes ethniques, clans et lignages.

Aucune des deux formes de fokonolo n'est démocratique : les anciens prennent les décisions à l'avance. Les « lilin-draza » (règles des ancêtres), qui prescrivent les rituels et les devoirs vis-à-vis des êtres surnaturels, des anciens et de la société, continuent de constituer la base de la gouvernance des Tanalana au sein du parc national de Tsimanampetsotsa et autour de celui-ci.

Dans leur essai intitulé « L'illusion participative », Blanc-Pamard et Fauroux (2004) soulignent la réalité non égalitaire des instances décisionnelles traditionnelles dans l'ouest de Madagascar, notant que leur fonctionnement est rarement démocratique et qu'il privilégie les personnes les plus riches et ayant un statut social élevé. Ils affirment également que les instances décisionnelles basées sur les lignages dans l'ouest de Madagascar se sont avérées mal adaptées à la gestion des questions qui s'étendent au-delà de la juridiction du lignage. En parallèle, les chefs de lignage ont reconnu qu'il était dans leur intérêt de mettre en avant la participation démocratique afin d'attirer les financements de bailleurs de fonds internationaux, ces derniers mettant l'accent sur la nécessité de processus de développement participatifs. Blanc-Pamard et Fauroux (2004) concluent qu'il y a un espoir que l'illusion participative se transforme en une participation significative à mesure que les villageois en plus grand nombre prennent l'habitude de participer activement aux réunions. 


\subsubsection{Les conflits fonciers et leur gestion}

Dans les communautés rurales malgaches, les causes des conflits fonciers sont, notamment, l'ambiguïté sur la propriété des terres au sein de la famille (Raison 1991), les disputes sur les mêmes espaces entre les occupants établis et les migrants récents (Fauroux 2001 ; Moizo 2001), et la concurrence entre les besoins liés à l'élevage et les besoins agricoles (Gezon 1999). Les conflits fonciers peuvent également se produire entre les communautés locales et l'État (Pollini 2007), entre les communautés et les ONG de conservation (Gezon 1997, 1999 ; Horning 2004) qui se sont souvent vu déléguer les pouvoirs de gérer les terres de l'État, ou entre les communautés et les investisseurs extérieurs (Medernach et Burnod 2013). Les conflits peuvent aussi diviser des villages entiers. À titre d'exemple, dans un village proche de la réserve spéciale d'Ambohitantely, un conflit sur les droits fonciers remontant à l'époque coloniale a divisé les villageois pendant des générations (Klein et al. 2007). Les conflits se produisent également entre des villages. À Marovoay, Medernach et Burnod (2013) décrivent un conflit qui a éclaté lorsque des investisseurs extérieurs n'ont pas pris la peine de consulter les villageois riverains Sakalava avant de négocier des concessions pour des plantations de jatropha avec les riziculteurs Betsileo. Les Sakalava étaient particulièrement révoltés, car cela touchait non seulement les terres où ils faisaient paître leur bétail, mais ils revendiquaient également des privilèges en qualité de famille fondatrice sur les terres occupées par les Betsileo, et estimaient donc qu'il leur revenait de négocier.

Aucune discussion sur les conflits fonciers à Madagascar ne serait complète sans mentionner le concept malgache de « fihavanana ». En effet, la société malgache accorde une grande valeur au « fihavanana $\rangle^{11}$, qui se traduit souvent par le terme de solidarité (Kneitz 2016). Le fihavanana met l'accent sur le maintien de relations pacifiques, tant avec les proches qu'avec les voisins (Kneitz 2016). Par conséquent, les Malgaches ruraux cherchent généralement à gérer d'abord les conflits sur les terres et les ressources naturelles au niveau de la famille ou du lignage (Gezon 1999 ; Di Roberto 2020). Si le conflit ne peut être résolu à ce niveau, ou s'il implique plusieurs familles ou lignages, les parties en conflit portent l'affaire devant le fokonolona, où les rey-mandreny font office de médiateurs et de juges (Gezon 1999). Une caractéristique importante de la résolution des conflits par le fokonolona est que celui-ci se concentre davantage sur la réconciliation des parties et la préservation des relations sociales, et moins sur l'attribution de la faute à une partie particulière (Rosés 2016).

La description par Gezon (1999) de la résolution des conflits au niveau du village près de la réserve spéciale d'Ankarana met en évidence l'importance que le fokonolona accorde à la réconciliation.

«Les règlements villageois fonctionnent sur la base de la coopération («fiarana ») et du consensus. D'après les anciens, l'objectif premier du fokonolona est d'établir des relations positives entre les parties au conflit en agissant comme un médiateur. Discerner le bien («ny mariny ») du mal est aussi important, et le fokonolona se donne souvent beaucoup de mal pour obtenir le plus de preuves possible. Mais, pour les anciens, mettre de l'ordre dans les relations (« mandaminy ») reste l'objectif premier souhaité » (Gezon 1999:86).

Ce n'est que si le fokonolona ne parvient pas à résoudre le conflit que les parties qui s'opposent peuvent porter l'affaire devant un tribunal d'État (Gezon 1999 ; Di Roberto 2020). Cependant, très peu d'affaires sont portées devant les juridictions de l'État (fanjakana). En effet, non seulement le fait de porter une affaire devant un tribunal d'État implique une dépense importante, mais les juges de ces tribunaux ne connaissent généralement pas l'historique des contentieux qui leur sont soumis

11 Le concept de fihavanana semble trouver ses origines dans la société Merina, mais il a été adopté comme principe social directeur dans une grande partie de Madagascar. On pense qu'il est dérivé de la racine du mot « havana », qui signifie « relation ». Un concept similaire, le « filongoa », se retrouve dans le sud de Madagascar chez les Bara (Scheidecker 2016). Néanmoins, Scheidecker rapporte que chez les Bara, « filongoa » s'applique de manière plus précise et englobe principalement la parenté patrilinéaire. 
(Gezon 1999; Di Roberto 2020). Tout aussi important, ces tribunaux ne fonctionnent pas sur la base du principe de la recherche du consensus ou de la coopération volontaire, deux éléments qui, aux yeux des malgaches ruraux, sont nécessaires à la légitimité (Kneitz 2016). Comme le souligne Sandron (2016), bien que l'utilisation du principe de fihavanana diminue en général la probabilité de vengeance par les parties lésées, elle est souvent loin d'être égalitaire en pratique, et tend à renforcer les inégalités sociales et les déséquilibres de pouvoir existants.

\subsection{Le Dina : lorsque le droit coutumier rencontre le droit écrit}

Une caractéristique clé des systèmes de gouvernance foncière traditionnelle à Madagascar est le recours généralisé aux dinas, ces codes de conduite qui régissent les interactions sociales au niveau local, notamment l'accès et l'utilisation des ressources naturelles. Bien que d'origine traditionnelle, les dinas sont considérés comme juridiquement valables par le droit écrit (Karpe et al. 2007). Karpe et al. (2007) décrivent les dinas comme des accords passés par les membres d'une communauté (c'est-à-dire le fokonolona) dans lesquels chaque personne indique son accord par un serment, et où des amendes ou des sanctions ( « vonodina ») sont envisagées ou appliquées à celles et ceux qui ne respectent pas les termes convenus. Traditionnellement, un dina est adopté par le fokonolona, puis consacré par un rituel. Aux yeux des participants, le rituel officialise le fait que le dina est non seulement un accord entre les membres de la communauté, mais également un pacte avec Zanahary (Dieu), les ancêtres et les esprits du lieu (Karpe et al. 2007). Les dinas étaient (et sont souvent encore) des accords oraux volontaires qui ont force de loi pour les membres de la communauté qui les décident. Cependant, de plus en plus, les fokonolona élaborent des dinas écrits, qui sont ensuite enregistrés soit auprès du fokontany, soit auprès de la commune (voir l'encadré 2). Les dinas sont le principal moyen par lequel les systèmes de gouvernance forestière traditionnelle à Madagascar ont réglementé l'accès et l'utilisation des ressources forestières (Fritz-Vietta 2015).
Encadré 2. Dina à Ambodilaingo (Commune de Beforona)

À Ambodilaingo, le conseil de village (composé des anciens de chacun des lignages présents dans le village) coopère avec les fonctionnaires de l'État pour créer des règlements locaux, appelés dinas. Bien que souvent non écrits, les dinas considérés très importants par la communauté sont rédigés et enregistrés à la commune. Cela place la communauté dans une position juridique beaucoup plus forte en cas de litige. Les dinas définissent les règles de conduite, les infractions ainsi que les peines en cas de violation des règles. Les exemples de comportements qui font l'objet de dina à Ambodilaingo incluent l'incendie volontaire, les vols de récoltes et les transgressions de tabous (Pollini 2007).

\subsection{Les droits fonciers traditionnels}

Les personnes considérées comme membres de la communauté, donc ayant des droits sur les terres et les ressources, ainsi que la nature de ces droits, varient selon le groupe ethnique et la situation géographique. En règle générale, les personnes qui appartiennent à des lignées, clans ou familles fondatrices, et qui restent dans la communauté, ont accès à la terre en vertu de leur appartenance au groupe fondateur (cf. Fauroux 2001 ; Moizo 2001 ; Di Roberto 2020). Les membres de la communauté peuvent acquérir des terres de diverses manières, notamment par succession, défrichement de terres inoccupées, métayage, dons intergénérationnels, emprunt, location et achat (Gezon 1997 ; Jones et al. 2018 ; Di Roberto 2020). Southall (1971:160) décrit les systèmes de parenté malgaches comme « des variantes d'un modèle cognatique, dans lequel la patrilinéarité a une certaine importance ». Il rapporte que la filiation patrilinéaire est plus courante parmi les groupes ethniques qui dépendent davantage de l'élevage extensif de bétail, comme les Sakalava et les Tsimihety, alors que la filiation bilatérale est plus courante parmi les groupes qui dépendent davantage de la culture du riz paddy, comme les Betsileo et les Merina. Le fait qu'un groupe 
organise sa filiation de manière patrilinéaire, matrilinéaire ou ambilinéaire affecte non seulement les personnes qui héritent des terres, mais aussi celles et ceux qui exercent un contrôle sur les terres du lignage et du clan.

À Bevary, près de la réserve spéciale d'Ankarana (Nord de Madagascar), les fils comme les filles des familles propriétaires héritent des droits d'utilisation des terres (Gezon 1997). Si les héritiers sont trop nombreux, ils héritent à tour de rôle. Gezon (1997) a rapporté que les locations de terres étaient courantes à Bevary et que, bien que rares, des terres pouvaient être vendues. Dans la région de Vakinankaratra (Hautes Terres du Centre), Di Roberto (2020) a documenté trois modes courants de transfert de droits fonciers à des proches : l'héritage, la donation et la délégation de droits. Les résidents de la région de Vakinankaratra avaient également accès à la terre par le biais du métayage, de la location et de l'achat. Cependant, les achats de terres sont relativement rares et se font le plus souvent entre membres d'une même famille. Bien qu'illégal, le métayage est un moyen courant permettant d'accéder à la terre dans de nombreuses régions (cf. Jarosz 1991; Evers 2001 ; Scales 2011). Bien que le métayage soit souvent considéré comme un moyen pour les sans-terre et les pauvres d'accéder à la terre, c'est aussi un moyen pour les agriculteurs plus aisés, qui peuvent se permettre d'embaucher de la main-d'œuvre pour travailler la terre, de produire davantage (Jarosz 1991).

Les migrants, que leur famille soit installée dans la région depuis des générations ou qu'elle soit arrivée récemment, doivent obtenir l'autorisation du « tompon-tany » pour défricher la terre (Fauroux 2001 ; Moizo 2001 ; Jones et al. 2018). Le processus permettant à un migrant d'obtenir cette approbation peut prendre un certain temps. Dans son étude des différents usages des forêts à Manongarivo (Nord de Madagascar), Ranjatson (2011) a décrit le long processus de légitimation sociale des migrants avant qu'ils ne soient autorisés à défricher une forêt. Pour avoir accès à la terre, un nouveau migrant devait d'abord emprunter des parcelles à une famille d'accueil et ne pouvait seulement cultiver des plantes annuelles comme le manioc ou le riz. Ce n'est qu'après plusieurs années passées à cultiver les parcelles empruntées que le « tompon-tany » l'autorisait à défricher sa propre terre.

À mesure que les terres se raréfient, les règles régissant l'accès des nouveaux arrivants à la terre peuvent changer. Thibaud et Colin (2010) ont rapporté qu'avant les années 1990, dans les communautés où les Masikoro sont prépondérants près de Toliara, les migrants de sexe masculin pouvaient facilement acquérir des terres, initialement par le biais d'accords de partage des cultures. S'ils se mariaient avec une personne de la communauté, ils pouvaient obtenir la permission de défricher la terre. Lorsque les migrants sont devenus plus aisés, ils ont commencé à acquérir des terres. Cependant, au milieu des années 1990, lorsque les terres sont devenues rares, les tompon-tany ont décidé, pour ralentir le rythme de défrichement, de faire payer aux migrants une taxe pour s'installer dans la région et défricher. Les migrants arrivés plus récemment ne peuvent plus obtenir la permission de défricher et n'ont accès à la terre que par le biais d'emprunts à court terme. Evers (2001 2006) documente une situation similaire en ce qui concerne les rizières de bas-fonds dans les Hautes Terres du Sud, où en 1967, les familles fondatrices (dont la plupart s'étaient installées dans la région dans les années 1950 et 1960) se sont réparti toutes les terres fertiles non réclamées et ont conclu un pacte selon lequel elles ne loueraient ni ne vendraient de terres aux nouveaux arrivants.

Il existe plusieurs moyens par lesquels les migrants peuvent s'intégrer socialement dans la communauté, les deux plus courants étant les cérémonies de fraternité de sang (« fatidra ») ou le mariage avec un membre de la communauté (Gezon 1997 ; Fauroux 2001 ; Jones et al. 2018 ; Di Roberto 2020). Cependant, la nature des droits accordés par ces relations varie. Ainsi, chez les Antakarana vivant près de la réserve spéciale d'Ankarana, dans le nord de Madagascar, les nouveaux arrivants qui passent par la « fatidra » acquièrent le droit d'accéder aux terres situées sur le territoire de la communauté. Cependant, ces liens ne vont pas jusqu'à accorder des droits de succession à la progéniture du nouveau venu (Gezon 1997). Chez les Sakalava de la région du Menabe, un migrant pouvait également intégrer la communauté par le mariage ou le « fatidra », mais ses droits sur la terre se limitaient aux droits d'usage, étaient toujours susceptibles d'être révoqués et le plaçaient dans une relation de dépendance à long terme vis-àvis du tompon-tany qui lui avait accordé ces droits (Fauroux 2001). 


\subsection{Les droits fonciers des femmes}

Malgré quelques lacunes importantes, les lois malgaches régissant les droits fonciers des femmes mettent l'accent sur l'égalité entre les sexes. L'article 6 de la Constitution de 2010 précise que « tous les individus sont égaux en droit et jouissent des mêmes libertés fondamentales protégées par la loi, sans discrimination fondée sur le sexe, le degré d'instruction, la fortune, l'origine, la croyance religieuse ou l'opinion ». De même, la loi sur les successions traite sur un pied d'égalité les conjoints survivants, hommes ou femmes ${ }^{12}$. Ainsi, qu'ils soient hommes ou femmes, les conjoints n'héritent pas l'un de l'autre, l'ordre de succession étant le suivant : enfants, petits-enfants, père et mère, frères et sœurs, oncles et tantes, cousins germains, puis conjoint survivant. Bien que les héritiers masculins et féminins héritent de manière égale, les cohéritiers peuvent convenir que les héritières recevront de l'argent plutôt que des terres. Selon la loi relative au mariage et aux régimes matrimoniaux de $2007^{13}$, les biens détenus en commun sont censés être divisés à parts égales entre le mari et la femme lors de la dissolution du mariage. Cependant, Widman et Hart (2019) ont noté que cette clause est rarement respectée et que, le plus souvent, le partage traditionnel (connu sous le nom de « kitay telo an-dalana ») attribue deux tiers au mari et un tiers à la femme.

En outre, la plupart des études que nous avons examinées n'ont fourni qu'une description sommaire des droits des femmes sur les ressources foncières ou forestières dans le cadre des systèmes de tenure coutumière. D'après le peu de données probantes disponibles, il semble que les droits des femmes à la terre varient considérablement selon le groupe ethnique. Mais, généralement, elles ont peu de droits. Cependant, dans certaines régions, comme les Hautes Terres du Centre, les droits des femmes semblent se renforcer face à l'évolution des conditions socio-économiques et à une prise de conscience croissante des lois du pays qui soutiennent l'égalité des sexes en matière de droits fonciers (Di Roberto 2020). Les exemples suivants, tirés de diverses régions de Madagascar, illustrent certains points communs et différences en matière de droits fonciers des femmes dans différents groupes ethniques.

\section{À Ambatomena (Région de Vakinankaratra), où les Merina constituent le groupe ethnique dominant, Di Roberto (2020) rapporte que, traditionnellement, seuls les hommes héritent des terres. Cependant, cet héritage s'accompagne de l'obligation de maintenir la terre dans la famille et de contribuer au rituel du « famadihana ${ }^{14}$, une activité généralement coûteuse. De plus, Di Roberto a noté qu'au fil du temps, les femmes d'Ambatomena sont de plus en plus susceptibles d'hériter de la terre. Les veuves d'Ambatomena conservent le pouvoir décisionnel sur les terres dont leurs maris ont hérité, ainsi que sur toutes les terres que le couple a acquises ensemble pendant le mariage.}

Toujours chez les Merina, dans un autre site de la région de Vakinankaratra, Valkonen (2018) a constaté que la succession était patrilinéaire et que les filles héritaient, le cas échéant, de moins de terres que les fils. Parfois, les filles recevaient de petites parcelles de la part de leurs parents. Cependant, comme les femmes n'héritaient pas ou ne recevaient que de petites parcelles, elles n'étaient pas tenues de contribuer aux festivités familiales, qui pouvaient être très coûteuses. Valkonen (2018) souligne que les règles n'étaient pas fixes et que certaines personnes donnaient les mêmes montants à tous leurs héritiers. La citation suivante de Valkonen (2018:182) illustre comment la satisfaction des prétentions foncières d'une personne dépend en grande partie de la nature des relations familiales existantes :

12 Loi n ${ }^{\circ} 68-012$ du 4 juillet 1968 relative aux successions, testaments et donations. Disponible à l'adresse suivante : http:// www.justice.mg/wp-content/uploads/textes/1TEXTES\%20NATIONAUX/DROIT\%20PRIVE/Textes\%20sur\%20la\%20 Famille/3.pdf.

13 Loi n²007-022 du 20 août 2007 relative au mariage et aux régimes matrimoniaux. Disponible à l'adresse suivante : http://jafbase.fr/docAfrique/Madagascar/LoiMariage.pdf.

14 Le Famadihana, souvent traduit par « le retournement des morts », est un rituel funéraire dans les Hautes Terres qui implique l'exhumation puis la réinhumation des corps dans le tombeau familial. Le rituel a lieu tous les 5, 7 ou 9 ans, et auquel participe l'ensemble ou une partie des descendants de l'ancêtre fondateur (Di Roberto 2020). 
«Les différences sociales existent, et des pressions peuvent être exercées sur les membres de la famille et leurs droits de tenure remis en question. Beaucoup de choses dépendent donc de la nature des relations. Si des tensions et des malentendus apparaissent, certains membres peuvent se voir retirer leurs parcelles ou voir leurs droits contestés, ce qui les place dans une situation d'insécurité. Les frères et sœurs qui ont quitté la zone (propriétaires absents), les enfants qui n'ont pas été officiellement reconnus comme descendants légitimes de leurs parents, les jeunes frères et sœurs et les femmes divorcées ou veuves sont particulièrement vulnérables ».

Parmi les Betsileo des Hautes Terres du Sud, Evers (2001) a constaté que la succession était patrilinéaire, les terres étant divisées de manière égale entre les fils. Ce n'est que dans de très rares cas que les femmes héritaient des terres à la mort de leurs parents. Cependant, dans les cas où la fille restait dans la région après son mariage (c'est-à-dire si elle épousait un homme de la région), elle pouvait avoir l'usage d'une partie des terres de ses parents si son mari ne possédait pas suffisamment de terres, ou si elle était mariée à un migrant.

\section{Pour les Bara vivant près du complexe forestier de Zombitse-Vohibasia (région d'Atsimo-} Andrefana), Moizo (2001) a rapporté que les rizières et les terres agricoles en altitude étaient transmises aux descendants de manière patrilinéaire, les femmes n'ayant que des droits d'accès et d'utilisation. Une femme pouvait posséder du bétail, mais lorsqu'elle quittait le village à son mariage, ses frères s'occupaient de ses bêtes ${ }^{15}$.

Parmi les Antemoro d'Analavory (région de Vatovavy Fitovinany), Rasoamalalavao et al. (2011) ont constaté que seuls les garçons pouvaient hériter des terres acquises par leurs parents par héritage. Cependant, les femmes pouvaient hériter des terres ancestrales (appelées « fatrange ») si elles ne se mariaient pas et restaient sur place. Si une femme était rejetée par son mari et retournait dans son village, les frères devaient lui fournir des terres. $\mathrm{Si}$, par la suite, elle se réconciliait avec son mari et retournait dans le village de celui-ci, les frères pouvaient reprendre les terres. Cependant, il convient de noter que la terre donnée aux femmes qui restaient au village et ne se mariaient pas était moins bonne que celle donnée aux hommes, et que les femmes n'en détenaient que l'usufruit.

Parmi les Sihanaka, près du lac Alaotra (région d'Alaotra-Mangoro), Jarosz (1991) a constaté que les femmes pouvaient hériter de terres, mais si elles déménageaient au moment de leur mariage, les hommes de leur famille restés dans leur village ancestral en devenaient métayers (Jarosz 1991). À la dissolution du mariage, les deux tiers de la terre que le couple avait acquise en commun étaient donnés au mari, la femme récupérant le tiers restant. Jarosz (1991) a remarqué que les femmes ayant accès à la terre avaient tendance à consacrer une plus grande partie de leur travail aux cultures maraîchères et sèches, tout en partageant leurs rizières irriguées avec les hommes..

Gezon (1997) a rapporté que chez les Antankarana du nord de Madagascar (région de Diana), les hommes et les femmes héritent tous de la terre. Sur les 59 femmes qu'elle a interrogées, $18 \%$ possédaient des terres. La plupart des femmes propriétaires terriennes étaient soit célibataires ou veuves. Bien que les femmes puissent posséder et possèdent du bétail, elles le confiaient à leur père ou à leurs frères pour qu'ils s'en occupent, car le soin du bétail est considéré comme une activité masculine. Gezon a souligné que la division sexuée du travail, culturellement construite, était un facteur qui limitait le contrôle des femmes sur les biens de production, tels que la terre, l'eau d'irrigation et le bétail. Les femmes sont fortement engagées dans le maraîchage, une activité sur laquelle elles exercent un contrôle. Le manque de contrôle des femmes sur les ressources productives était exacerbé par leur engagement limité dans les instances décisionnelles clés. Cependant, tout comme Valkonen (2018), Gezon a noté que les femmes qui avaient des liens sociaux avec des familles puissantes pouvaient exercer une influence substantielle sur les processus décisionnels villageois et familiaux. Elle a également constaté que si les femmes ne prenaient généralement pas la parole lors

15 Il faut noter que le principe directeur était que le bétail restait au village, quel que soit le sexe du propriétaire. Si un homme Bara choisissait de vivre ailleurs, son bétail restait également au village et était pris en charge par un parent masculin (Moizo 2001). 
des réunions du fokonolona, certaines d'entre elles étaient capables d'influencer les décisions en coulisse par le biais de commentaires faits en aparté aux anciens ou aux enfants de sexe masculin. Gezon (2002:691) conclut ainsi que « les femmes utilisent de manière proactive les systèmes locaux et régionaux de résolution des conflits pour garantir leur accès continu aux ressources et leur capacité à les façonner et à les transformer ».

\subsection{Les catégories locales d'utilisation des terres et les droits fonciers correspondants}

Les droits de tenure varient en fonction de la catégorie d'utilisation des terres. Ici, nous donnerons des exemples de la variété des droits de tenure associés aux jardins et aux parcelles résidentielles, aux rizières inondées de façon saisonnière, aux terres agricoles en altitude, aux pâturages et aux forêts.

\subsubsection{Les jardins et les habitations}

Parmi les études incluses dans notre examen, peu d'entre elles ont abordé les modalités de tenure pour les jardins et les habitations. Lorsque c'était le cas, les droits de tenure décrits ressemblaient aux droits de propriété privée détenus au niveau de la famille nucléaire (McConnell 2002 ; Pollini 2007 ; Thielsen 2016). McConnell (2002) rapporte qu'à Vohibazaha, les jardins (appelés là-bas « tanimboly ») étaient situés au fond des vallées, près des habitations, et étaient exploités selon des pratiques de culture intensive. Dans cette région, une distinction est faite entre « tanimboly » et " potro ${ }^{16}$, ce dernier étant un jardin dans lequel on a construit de petites cabanes. Les droits fonciers sur le « potro » sont plus importants que ceux sur le «tanimboly », car la présence d'une cabane implique que l'utilisateur cultive la terre en permanence. La plantation d'arbres et la construction de cabanes renforcent les revendications foncières. Les arbres plantés dans les jardins indiquent également que l'utilisateur possède des droits permanents et exclusifs (Pollini 2007).

\subsubsection{Les terres agricoles}

Les droits de propriété sur les terres agricoles sont généralement détenus par des lignées ou des clans. Cependant, dans les zones où les terres adaptées à l'agriculture, particulièrement la riziculture, sont rares, ils peuvent être détenus (et gérés) par des familles nucléaires ou des ménages (cf. Pollini 2007 ; Di Roberto 2020). La possession de titres fonciers délivrés par l'État est rare, et plusieurs études ont indiqué que les titres n'étaient pas nécessaires pour que les revendications foncières soient reconnues localement comme légitimes (Spack 2005 ; Cullman 2015 ; Valkonen 2018 ; Di Roberto 2020). Depuis 2005, les certificats fonciers sont plus couramment délivrés que les titres, mais ils sont eux aussi relativement rares. En utilisant les données d'une enquête menée dans quatre régions en 2015, Di Roberto (2020) a calculé le pourcentage de parcelles détenues avec des certificats fonciers. Ce pourcentage variait d'un minimum de $4 \%$ dans la région d'Analamanga (Hautes Terres du Centre) à un maximum de $19 \%$ dans la région de Diana (Nord de Madagascar). Dans de nombreuses régions, comme la zone de Mivili dans le nord-ouest de Madagascar (près de Marovoay dans la région de Boeny), il n'y avait pas de guichet foncier local auprès duquel demander un certificat foncier (Medernach et Burnod 2013).

Dans l'ensemble du pays, les terres les plus fertiles se trouvent généralement dans les plaines avec inondation saisonnière, souvent appelées « baiboho », qui sont dédiées à la production de riz pendant la saison humide et à des cultures comme les tomates ou les oignons pendant la saison sèche (McLain et al. 2019). Dans les zones où ces plaines inondées de façon saisonnière sont rares, ces terres sont

16 Messerli (2003) rapporte qu’à Beforona, qui est également situé près du parc national de Mantadia, le terme « potro » désigne la cabane, plutôt que le jardin dans lequel il se trouve. De telles cabanes peuvent se trouver sur des terres utilisées pour des cultures itinérantes ( tavy ») ainsi que dans des jardins («tanimboly »). L'emplacement du « potro » change d'une saison de tavy à l'autre, car les jachères ne peuvent pas être utilisées de façon permanente. 
surtout la propriété privée de familles (cf. Di Roberto 2020 ; Klein et al. 2007 ; Pollini 2007). Dans les zones périphériques de la réserve spéciale d'Ambohitantely, située à la limite orientale des Hautes Terres du Centre, les fonds de vallée où se trouvent les rizières couvrent une superficie relativement peu importante et sont soumis à des droits coutumiers hérités au sein de familles établies («tarikas ») qui les exploitent collectivement (Klein et al. 2007). Les nouveaux arrivants dans cette région sont généralement des métayers, qui dépendent des familles établies pour accéder à la terre. Dans les zones où les pasteurs sont historiquement nombreux, comme le plateau Mahafaly et les zones côtières adjacentes, la région du Menabe et la région Boeny, les tompon-tany des sociétés pastorales occupant ces zones ont initialement donné aux immigrants, notamment aux Betsileo, le droit d'utiliser les « baiboho » situés sur leurs territoires pour cultiver le riz (Fauroux 2001 ; Medernach et Burnod 2013 ; Moizo 2001).

Les collines adaptées aux cultures sèches sont appelées « tanety $\|^{17}$. Dans les régions où la terre est encore abondante, les droits sur les « tanety » sont généralement obtenus en défrichant la terre et en la cultivant (Klein et al. 2007). Dans de nombreuses régions, les « tanety » ont moins de valeur que les plaines inondées de façon saisonnière. Toutefois, l'exploitation des « tanety » peut être lucrative en fonction de la demande du marché pour les produits cultivés, de la proximité des marchés et des infrastructures de transport. Les ménages cultivent souvent leurs champs de « tanety » séparément plutôt que collectivement.

Dans la communauté de Vohibazaha, près du parc national de Mantadia, les rizières sont regroupées autour des tombeaux familiaux dans la montagne, par lignage (McConnell 2002). Les emprunts et les prêts de terres sont monnaie courante au sein de la communauté, ainsi que des échanges de travail réciproques. Ici, les rizières des Hautes Terres sont mises en jachère pour qu'elles retrouvent leur fertilité, et les droits d'utilisation de ces champs reviennent à perpétuité au fermier qui a été le premier à les défricher. Les peuplements d'arbres situés sur les terres en jachère sont considérés comme « fady » (tabou) et il est interdit d'y toucher (McConnell 2002). Ranjatson, co-auteur de ce rapport, a constaté que l'exploitation des arbres verts des jachères était interdite à Beforona, qui est situé près du parc national de Mantadia. Cependant, les arbres morts sur pied («tsangamaina ») étaient en accès libre. Les familles propriétaires des terres en jachère pouvaient couper des arbres vivants, mais uniquement si elles étaient à court de bois de chauffe, une pratique connue sous le nom de « mandazo », qui signifie « faire disparaître les arbres verts ». Les arbres coupés eux-mêmes sont appelés « lazo » (arbre fané).

Dans les villages qui manquent de terres, la plupart des terres situées le long des rivières ont déjà été distribuées aux familles (Gingembre 2017). Dans ces zones, les Hautes Terres («tanety ») sont converties en champs pour répondre aux besoins fonciers de la jeune génération, diminuant ainsi les terres disponibles pour les pâturages et augmentant la probabilité de conflits entre éleveurs et agriculteurs.

\subsubsection{Les terres de pâturage}

La plupart des études comportant des détails sur les droits aux terres de pâturage se sont concentrées sur le plateau de Mahafaly (Moizo 2001 ; Goetter et Neudert 2016 ; Thielsen 2016), la région d'Atsimo Andrefana (Horning 2004) et la région du Menabe (Fauroux 2001, Scales 2011). Dans ces régions, les pâturages sont généralement gérés comme un bien commun, mais avec des zones réservées aux troupeaux de certaines familles ou lignées. Les pâturages sont généralement situés sur les Hautes Terres, où des cultures sèches peuvent également pousser. Les éleveurs de bétail utilisent différentes zones au sein des pâturages communs à des fins différentes, et ces zones ont différents types de tenure. La description des Bara dans la région d'Atsimo Andrefana faite par Horning (2004:84) illustre la façon dont l'utilisation des terres, l'organisation sociale et la tenure sont imbriquées :

17 Dans certaines parties du nord-ouest de Madagascar, les terres arides sont connues sous le terme de « tanimboly » (McLain et al. 2019). 
«Si l'on observe les territoires des villages Bara, il devient évident que le pastoralisme dicte les modalités de l'organisation spatiale. En d'autres termes, les cellules spatiales sont organisées autour des besoins du bétail. À l'intérieur de chaque village (« tanana » ou « tana $»)$, il existe des zones désignées pour le parcage des zébus pendant la nuit (« vaia »). En dehors du cercle (imaginaire) des établissements humains, et au-delà des champs agricoles (« tanimboly »), de vastes zones de savanes (« monto »), de forêts (« ala »), de rivières (où les zébus s'abreuvent et barbotent) et de forêts galeries («sakasaka ») et les sommets des collines constituent ensemble l'espace ou l'unité de pâturage.

La zone de savane située immédiatement à l'extérieur du village (à environ un kilomètre) est généralement désignée comme un pâturage collectif. Au-delà de cette zone, chaque lignage dispose d'une zone réservée où il emmène le bétail de ses membres. Certaines de ces zones se trouvent à l'intérieur des forêts. Les « toets 'aomby », qui sont des campements temporaires où les membres d'un lignage gardent le bétail familial, sont situés entre les villages et les forêts. Bien que cela ne soit pas explicitement dit, ces campements servent de postes de surveillance pour observer la circulation sur le territoire du village et dans la forêt.

L'organisation physique des zones de pâturage est intimement liée à l'organisation sociale, chaque lignage parquant ses troupeaux et contrôlant l'espace sur lequel les troupeaux respectifs paissent. Le territoire du village peut donc être représenté comme un ensemble de zones lignagères, claniques ou ethniques dans lesquelles chaque espace linéaire est organisé de manière prévisible : il existe des zones spécifiques où les zébus sont parqués (« vaia »), où ils sont rassemblés ( « kija »), où ils broutent, s'abreuvent, barbotent et se reposent. Enfin, il existe des sentiers spécifiques où ils circulent, sont examinés et comptés («kizo »)».

Chez les Bara du plateau d'Ihorombe (Gingembre 2017), les villages fondateurs détiennent des droits collectifs (de gestion, d'exclusion et d'attribution) sur les parcours communaux (« tanypokonolona ») et fixent les règles d'utilisation pour les membres des autres villages (typiquement des droits d'accès et d'usage). L'utilisation de ce territoire s'accompagne souvent d'obligations d'assistance et de réciprocité. De plus, une surveillance collective contre le vol de bétail est pratiquée dans cette zone. Chaque village dont les terres ont été traversées par des voleurs a le devoir de surveiller le bétail. Ainsi, un village qui ne peut pas identifier le lieu exact d'où est parti le bétail volé doit payer une compensation au propriétaire de celui-ci.

Lorsque des pâturages considérés comme un bien commun sont convertis en terres agricoles, des conflits surgissent parfois entre éleveurs et agriculteurs. Cela a été le cas à Bevary, près de la réserve d'Ankarana dans le nord de Madagascar, où le fokonolona a autorisé un agriculteur à creuser un canal et à défricher un champ dans une partie où les pâturages sont gérés en commun (Gezon 1999). Bien que les pâturages de Bevary ne soient pas privés, les habitants ont reconnu que certains troupeaux avaient le droit de paitre dans des parties spécifiques des terres communes. Le bétail qui avait l'habitude de paître dans cette zone a continué à le faire, causant des dommages aux cultures de l'agriculteur. Finalement, l'affaire a été portée devant le fokonolona, qui a décidé que l'agriculteur devait clôturer ses terres pour empêcher de futures intrusions.

Il y a 20 à 30 ans, dans certaines régions du sud-ouest de Madagascar, les habitants ont commencé à réclamer la privatisation des droits en réponse à la pénurie d'arbres fourragers appelés « samatas » (Goetter et Neudert 2016 ; Thielsen 2016). L'encadré 3 décrit l'éventail des droits qui existent désormais pour les « samatas ». Malgré les nombreuses tentatives de restriction au niveau local et de l'État, ces enclos de privatisation sont largement acceptés par les locaux, qui en comprennent la motivation étant donné la rareté des arbres samatas et leur pratique culturelle consistant à permettre aux membres de la communauté de revendiquer des terres ouvertes (Goetter et Neudert 2016). Néanmoins, les membres de la communauté craignent que des conflits ne surgissent avec les « hommes forts » qui ont été les plus actifs dans le balisage des enclos privés. 


\section{Encadré 3. Privatisation d'une ressource commune}

Sur le plateau Mahafaly, dans le sud-ouest de Madagascar, Goetter et Neudert (2016) ont constaté que chez les Tanalana ${ }^{11}$, des droits privés sur l'Euphorbia stenoclada existaient aux côtés d'un libre accès aux zones de pâturage communautaires. L'Euphorbia stenoclada est un petit arbre succulent utilisé pour le fourrage du bétail et est désigné localement sous le nom de « samata ». L'accès aux samatas a toujours été ouvert à tous, à l'exception des samatas situés près des enclos à bestiaux privés. Cependant, la réduction des précipitations, la conversion généralisée des pâturages de samatas en terres agricoles et les techniques destructives de récolte de samatas, ont réduit le nombre de ces arbres, suscitant la privatisation de cette ressource naturelle importante.

Goetter et Neudert (2016) ont recensé trois types de droits sur les arbres samatas :

1. Samatas balisés : Il s'agit d'arbres situés dans des zones de pâturage communautaires, mais qui ont été balisés et revendiqués comme propriété privée à l'aide de rangées de cactus. Les droits portent sur les arbres mais pas sur la terre.

2. Samatas autour des enclos à bestiaux : il s'agit d'arbres entourant des enclos privés situés dans des zones de pâturage communes. Les droits comprennent le droit d'utiliser les arbres pour le fourrage et de vendre des arbres samatas, mais pas de droits sur la terre.

3. Enclos de samatas : Il s'agit d'arbres qui sont entourés de clôtures et qui sont parfois libres d'être utilisés par l'ensemble des membres du clan. Les droits comprennent les droits sur la terre et sur les arbres.

Les conflits concernant la distance acceptable d'utilisation privée autour de l'enclos du bétail et la taille des enclos ont été débattus à différents niveaux (fokontany, commune, clan) depuis trois décennies. Néanmoins, jusqu'à présent, aucune solution n'a été trouvée (Thielsen 2016).

1 Les Tanalana sont un sous-groupe des Mahafaly.

\subsubsection{La tenure forestière}

Dans les régions de Madagascar où les systèmes de tenure coutumière prédominent, les forêts ont des valeurs et des significations multiples. En effet, les forêts fournissent des ressources de subsistance (bois de chauffe, matériaux de construction, nourriture, gibier), fonctionnent comme une réserve foncière pour les générations futures et sont des sites où résident les esprits et les ancêtres (Scales 2012 ; Cullman 2015). Les forêts, qu'il s'agisse de forêts-galeries, de forêts secondaires ou de savanes boisées, sont aussi sans doute les paysages dans lesquels les systèmes de tenure coutumière sont les plus susceptibles d'être fortement impactés par le système de tenure étatique.

La loi foncière de 2005 dispose que les terres occupées ou mises en production sont présumées être des propriétés privées, et donc que les droits coutumiers sont reconnus sur la plupart des terres agricoles et des espaces où se trouvent des habitations. Cependant, même si de nombreuses communautés rurales ont des revendications coutumières de longue date sur les forêts ou les pâturages avoisinants, ces terres ne sont pas considérées comme occupées ni comme ayant été mises en production (Aubert et al. 2013).

Par conséquent, la plupart des terres forestières (et des pâturages) sont soit supposées faire partie du domaine public de l'État, soit, si les procédures appropriées ont été suivies, ont un statut juridique spécifique, comme un parc national, une réserve naturelle ou un autre type d'aire protégée. Cependant, dans la mesure où la capacité de l'État à gérer les terres forestières reste limitée, en pratique, les règles de tenure coutumière continuent de jouer un rôle important dans l'accès et l'utilisation des forêts dans la plupart des zones rurales de Madagascar. 
Depuis la promulgation de la loi GELOSE en 1995 et la promulgation du décret ultérieur de 2001 simplifiant le processus de cogestion, les contrats de gestion forestière (CGF) sont devenus le principal moyen permettant aux communautés d'exercer légalement leurs droits sur les forêts et les parcours qui se trouvent sur leur territoire (Aubert et al. 2013). En vertu de la loi ${ }^{\circ}{ }^{2015-005}$ portant refonte du Code de Gestion des Aires Protégées, les communautés peuvent utiliser des contrats de cogestion pour acquérir des droits de gestion reconnus par l'État sur les aires protégées. L'article 42 de cette loi précise également que les règles de gestion doivent faire prévaloir le « respect des normes et pratiques traditionnelles (dina, fady, lieux forestiers sacrés, aquatiques ou autres) observées par les communautés locales concernées ».

Les modalités d'attribution des droits sur les terres et les ressources forestières dans le cadre des systèmes traditionnels varient à travers Madagascar, tout comme la capacité des communautés à faire respecter leurs règles.

Dans le nord du pays, dans le parc naturel de Makira, où les habitants sont des Betsimisaraka et des Tsimihety, Cullman (2015) a constaté que la plupart des terres forestières étaient attribuées dans le cadre du système traditionnel à des ménages spécifiques ou à de grandes familles étendues, plutôt que d'être traitées comme un bien commun à l'échelle de la communauté ou une ressource en accès libre. Par conséquent, lorsqu'une ONG internationale de conservation a cherché à mettre en œuvre un contrat de gestion forestière (CGF) pour la zone tampon autour du parc avec les communautés locales en partant du principe que la forêt était gérée de manière communautaire, des tensions sont rapidement apparues, car les individus avaient le sentiment que leurs droits n'étaient pas respectés. En outre, pour signaler que le parc lui-même était interdit d'utilisation, l'ONG a déclaré le parc comme étant une forêt taboue/sacrée ( «alafady »). Cela a également provoqué la colère des habitants qui ont estimé que l’ONG avait usurpé les pouvoirs détenus par leurs chefs traditionnels («tangalamena »).

Elmqvist et al. (2007) ont examiné les règles d'accès aux ressources forestières et leur application dans quatre zones de la région d'Androy, dans le sud-est de Madagascar. Dans les quatre sites, le contrôle de l'État était effectivement inexistant, ce qui a eu pour conséquence la prédominance des règles coutumières ou, dans certains cas, l'absence de systèmes de tenure forestière étatiques ou traditionnelles. Dans l'ouest de l'Androy, région qui a connu une longue histoire d'exode vers d'autres régions de Madagascar, le système de tenure coutumière n'existe plus, la forêt devenant une ressource en accès libre :

« Selon les chefs locaux, une pratique coutumière est utilisée pour revendiquer des terres, en utilisant des figuiers de Barbarie (Opuntia spp) pour marquer les nouvelles frontières, mais les mécanismes institutionnels qui réglementent cette pratique ne sont pas employés, car les anciens parmi les nouveaux habitants n'ont pas d'autorité établie sur la terre. En pratique, on assiste à une situation d'accès libre, avec une application insuffisante des règles officielles et un dysfonctionnement du système coutumier » (Elmqvist et al. 2007:7).

En revanche, les systèmes de tenure coutumière ont continué à fonctionner correctement dans le nord et le centre de l'Androy. Elmqvist et al. (2007) ont rapporté que les nouveaux colons voulant s'installer de façon permanente dans la région doivent passer par les autorités claniques pour obtenir l'accès à la terre, et que quiconque souhaite couper un arbre doit obtenir l'approbation du fokonolona, ainsi que de l’État (« fanjakana »).

Cependant, il est difficile d'appliquer la loi concernant les personnes venant de l'extérieur dans ces deux sites en raison de la faible densité de population et des difficultés qui en découlent pour contrôler l'utilisation forestière. Dans le sud de l'Androy, où la densité de population est plus élevée, le système traditionnel continue également de fonctionner. Toutes les parcelles forestières ont été attribuées à des clans ou des lignées, et certaines parcelles sont désignées comme forêts sacrées, ce qui restreint l'accès à certaines personnes et la récolte à certaines ressources. Il est important de noter qu'Elmqvist et al. (2007) ont constaté une relation positive entre la force du système traditionnel et la couverture forestière. En effet, la plus grande réduction de la couverture forestière s'est produite dans l'ouest de 
l'Androy, là où le système coutumier s'était effondré et où un système d'accès libre s'est développé. A contrario, dans les sites où les systèmes traditionnels ont continué à bien fonctionner, la couverture forestière est restée stable.

L'étude de Horning de 2004 sur les règles en usage dans quatre forêts en Ibara offre un aperçu instructif des différences concernant le respect des règles selon que les règles forestières sont principalement issues de la communauté ou imposées par l'État. Les habitants des villages situés à proximité de ces forêts exploitent les ressources forestières soit pour leur propre usage (par exemple, bois de construction et de chauffe, nourriture, médicaments), soit, dans une moindre mesure, pour la vente commerciale (par exemple, charbon de bois, bois d'œuvre, minéraux) ${ }^{18}$. Les règles communautaires étaient moins restrictives pour les ressources alimentaires et plus restrictives pour la récolte commerciale de bois mort ou vivant. Horning (2004) a identifié deux types distincts de situations de tenure forestière parmi les communautés forestières étudiées.

1. Communautés où les règles locales protègent bien les forêts. La protection des forêts était forte dans les communautés les plus éloignées et qui vivent près de l'aire protégée Analavelona. Les membres de la communauté considéraient la forêt comme la demeure sacrée des ancêtres de leurs clans fondateurs; ils l'appréciaient également comme un endroit où ils pouvaient dissimuler leur bétail afin d'éviter de payer des taxes. Dans les communautés les plus reculées, les règles forestières étaient fixées par les chefs du lignage ou du clan, et étaient généralement respectées. Le risque d'être pris en infraction était élevé, car les bergers locaux situés dans des campements de bergers à la lisière de la forêt pouvaient facilement surveiller la zone. De nombreux habitants croyaient qu'ils recevraient une punition divine (maladie ou mort) s'ils enfreignaient les règles. Les membres de la communauté étaient moins enclins à respecter les règles de l'État, qu'ils jugeaient comme étant trop restrictives et ne reflétant pas les valeurs locales, et que l'État n'avait pas la capacité de faire respecter. L'efficacité des règles locales s'est reflétée sur le plan écologique : une analyse de l'imagerie satellitaire menée entre 1989 et 2004 n'a révélé aucun changement détectable au niveau de la couverture forestière.

2. Les communautés où les règles locales protègent mal les forêts. Dans les communautés plus éloignées de la forêt, et où de nombreux migrants ou plusieurs groupes ethniques étaient présents, l'utilisation de la forêt était davantage fondée sur les règles de l'État que sur celles de la communauté. Ces communautés étaient socialement moins soudées et les autorités traditionnelles avaient moins de pouvoir que dans les communautés où la protection des forêts était forte. Les forêts situées à proximité de ces communautés ont souffert d'une déforestation importante et de l'utilisation illégale de la forêt, comme la pratique du « tavy » pour défricher les champs et la récolte commerciale de bois de charpente, de bois de chauffe et de charbon de bois. L'exploitation commerciale était facilitée par l'accès facile aux marchés locaux. La gestion de l'État dans ces régions mettait l'accent sur la protection de la forêt au détriment des moyens de subsistance locaux, ce qui, d'après Horning, pourrait expliquer la forte incidence de l'exploitation forestière illégale.

Les forêts et arbres sacrés sont une autre caractéristique importante des systèmes de tenure coutumière à Madagascar. L'accès et l'utilisation de ces forêts sont soumis à des règles différentes de celles appliquées aux autres zones forestières. L'encadré 4 décrit des exemples de forêts sacrées chez les Sakalava, les Tanalana et les Betsimisaraka. Sur la base de travaux menés dans la région d'Androy, Tengö et al. (2007:689) soutiennent que « les croyances en leurs ancêtres et leur influence sur la vie quotidienne» font partie intégrante des systèmes locaux de gouvernance forestière. Ils suggèrent que les stratégies de conservation devraient mettre l'accent sur la compréhension de ces croyances pour s'appuyer sur cellesci, plutôt que sur l'utilisation d'approches qui les sapent, comme l'imposition de systèmes étatiques trop rigides et basés sur des valeurs étrangères aux membres des communautés locales.

18 L'exploitation forestière à grande échelle est plus susceptible de se produire dans les zones où des investisseurs extérieurs sont impliqués dans l'extraction des ressources (Horning 2004 ; Pollini 2007). 


\section{Encadré 4. Exemples de règles relatives aux forêts sacrées à Madagascar}

Les Sakalava du Menabe central (ouest de Madagascar) distinguent la forêt proche des villages (« ala »), la grande forêt (« ala be ») située à une certaine distance des zones habitées, et la forêt sacrée, appelée en malgache « ala fady » (Scales 2012) ${ }^{1}$. La collecte de bois-énergie et le défrichage de la forêt sont interdits dans la forêt sacrée, et il y a des zones où même le passage n'est pas autorisé. La plupart des tabous sont liés aux liens avec les ancêtres puisque les tombeaux familiaux sont souvent situés dans les forêts sacrées (Scales 2012). Les grands arbres anciens, notamment le tamarinier ( « kily » en malgache ou Tamarindus indica) et le baobab de Grandidier (« renala » en malgache ou Adansonia grandidieri), sont souvent le lieu des offrandes aux esprits. Les fady concernant l'utilisation de la forêt s'appliquent à la fois aux « zana-tany » (personnes nées localement, littéralement les personnes de la terre) et aux « vahiny » (personnes venues de l'extérieur).

Les communautés Tanalana au sein et autour du parc national de Tsimanampetsotsa dans le sudouest de Madagascar ont désigné certaines zones comme sacrées (Thielsen 2016). Les faly (tabous) locaux interdisent l'utilisation de certaines espèces de plantes et d'animaux, ainsi que des zones désignées comme sacrées. Par exemple, il est interdit de couper des arbres autour des tombes. Par ailleurs, les anciens et les êtres surnaturels sont chargés de veiller au respect des règles ancestrales (« lilin-draza »), notamment les faly. Les tabous liés aux lieux s'appliquent aussi bien aux personnes extérieures qu'aux membres de la communauté. Cependant, au fil du temps, l'adhésion au « lilin-draza » a diminué, tant chez les membres de la communauté que chez les personnes extérieures. Thielsen (2016) attribue le déclin de la légitimité des autorités traditionnelles dans la région à l'abandon des croyances religieuses traditionnelles au profit du christianisme parmi les membres de la communauté, à l'augmentation de l'immigration de personnes n'ayant pas de liens de parenté avec les villageois locaux et au manque d'autres sources de revenus.

Parmi les Betsimisaraka, près du parc national de Mantadia dans l'est de Madagascar, les tombes ancestrales des premiers colons sont situées sur des crêtes de montagnes couvertes de forêts (McConnell 2002). La zone entourant les tombes est appelée « sembontrano », et la conversion des terres forestières " sembontrano » en champs est étroitement contrôlée par le patriarche, chef du clan appelé « tangalamena » en malgache (McConnell 2002). Le sacrifice d'un zébu est nécessaire avant que le « sembontrano » puisse être utilisé pour la culture du riz pluvial, et une fois les nutriments épuisés, la zone doit être laissée en jachère pendant au moins huit ans. La zone adjacente au « sembontrano » est appelée « jingeranto ». Bien que l'accès au « jingeranto » soit moins strictement contrôlé, ces terres comportent quelques arbres disséminés considérés comme tabous («fady »), et qui ne peuvent pas être utilisés (McConnell 2002).

Parmi les Antandroy vivant dans la région d'Androy, dans le sud de Madagascar, Tengö et al. (2007) ont documenté plusieurs catégories de parcelles de forêt sacrées, chacune disposant de son propre ensemble de règles. Il s'agit de :

1. parcelles de forêt avec des tombes (« ala kibory »), où l'accès était strictement réglementé et où un sacrifice de zébu était requis avant toute récolte de ressources ;

2. parcelles de forêt sans tombes ( « salata ») où l'on dépose les morts en attendant de les enterrer. Bien que les personnes et le bétail puissent entrer dans ces zones, aucune ressource ne pouvait y être récoltée ;

3. bosquets de miel (« ala fano-hofa ») étaient dédiés à la production de miel. Les arbres de ces bosquets ne pouvaient pas être coupés, mais le miel et le bois mort pouvaient être récoltés ;

4. les parcelles de forêt avec des esprits ; il s'agissait de parcelles de forêt ancienne et considérées comme extrêmement dangereuses ;

5. les forêts privées ; les propriétaires pouvaient récolter des arbres vivants et d'autres ressources ou accorder à d'autres la permission de le faire ;

6. les parcelles de forêt dédiées aux cérémonies ; généralement, seuls les guérisseurs et les devins avaient accès à ces zones ;

7. les parcelles de forêt commémorative ; il s'agissait de zones qui avaient été des territoires royaux, des fosses communes et des champs de bataille. L'application des règles relatives aux forêts sacrées incombait au clan propriétaire de la parcelle de forêt.

1 Dans sa monographie des Sakalava dans la région du Menabe, Goedefroit rapporte que les habitants font également une distinction entre l'« ala be » (grande forêt) et l'« ala mandiniky » (petite forêt dégradée). 
L'étude de cas d'Andriamalala et Gardner (2010) sur le dina de Velondriake, une grande aire marine protégée dans le sud-ouest de Madagascar impliquant plusieurs fokontany, fournit un aperçu instructif du processus de création du dina de cogestion, ainsi que certains des défis associés à l'application de ces dinas. Pour l'aire marine protégée de Velondriake, la communauté de base (VOI), qui était composée des membres des 24 fokontany situés à l'intérieur ou à proximité de l'aire marine protégée, a commencé par organiser plusieurs réunions pour parvenir à un consensus sur les règles à inclure dans le dina. Ceci a pu être réalisé grâce à l'aide de plusieurs ONG externes de conservation bénéficiant de solides soutiens financiers. Le chef du fokontany (désigné comme le parrain de la communauté de base) et le maire de la commune ont signé le dina. Dès signature par le maire, le dina est entré en vigueur au niveau local.

Cependant, pour être valide aux yeux de l'État, et donc opposable aux personnes extérieures, le dina doit être conforme à la Constitution, aux lois et aux règlements malgaches. Au cours de la première étape de ce processus, appelée homologation, le chef de district et les autorités du Ministère régional concerné examinent et approuvent le dina. À la dernière étape du processus, la communauté de base a présenté le dina au président du tribunal afin que ce dernier vérifie la conformité du document aux lois nationales. L'approbation du tribunal obtenue, la communauté de base se trouve dans une position beaucoup plus forte dans l'hypothèse où des conflits surviendraient lors de l'exécution. Après l'approbation du dina, chaque village a reçu une copie de la lettre d'homologation. Le dina de Velondirake précise les règles régissant l'utilisation des ressources dans l'aire marine protégée, et prévoit aussi des sanctions (appelées « vonodina ») en cas d'infraction. Les vonodina comprennent des amendes ainsi que la saisie des produits récoltés illégalement.

Parmi les défis associés à la mise en œuvre du dina de Velondriake, on peut citer :

1. la réticence à infliger des amendes aux autres membres de la communauté ;

2. le manque de représentativité de certains sous-groupes (c'est-à-dire les femmes, les jeunes et les migrants) au cours du processus décisionnel, et leur opposition et résistance ultérieures aux règles ;

3. le conflit entre les normes culturelles et les lois nationales (concernant par exemple la récolte des tortues de mer);

4. des amendes trop élevées par rapport aux moyens dont disposent les membres de la communauté ;

5. une connaissance limitée du dina et de sa composition par de nombreux membres de la communauté.

L'analyse menée par Bérard en 2011 de 32 dinas élaborés pour des contrats de gestion forestière dans le cadre de la loi GELOSE a révélé des problèmes similaires. Le nouveau type de dina avait tendance à suivre un modèle commun, élaboré par des organisations internationales de conservation, qui ne tenait compte ni de la réglementation traditionnelle de l'utilisation des forêts en vigueur dans les communautés, ni des réalités sur le terrain. Le nouveau dina mettait l'accent sur les interdictions et les amendes associées aux violations des règles, une approche qui, comme le souligne Bérard, est en opposition directe avec le dina traditionnel, qui privilégie la cohésion sociale à la répression. Les membres de la communauté respectent peu ces dinas « vazaha ». Dans la mesure où la loi GELOSE précise que les dinas ne sont applicables qu'aux membres des communautés qui les élaborent, la répression des infractions commises par des personnes venues de l'extérieur est problématique pour les autorités communautaires. Bérard attribue l'écart entre les nouveaux dinas et ce qui est réellement nécessaire sur le terrain à une vision désuète de la loi, dans laquelle l'État recherche l'uniformité et promeut une approche contractuelle, alors qu'il est indispensable de mieux comprendre les conceptions locales de la loi forestière et les stratégies compatibles avec celles-ci.

\subsection{Les principaux facteurs de changement dans l'utilisation des terres et les systèmes de tenure coutumière}

Notre étude a permis d'identifier plusieurs facteurs clés de changement dans l'utilisation des terres et les systèmes de tenure coutumière à Madagascar. 
1. Les politiques et les lois nationales qui ne reconnaissent pas les revendications coutumières sur les terres dites « inoccupées », comme les pâturages et les forêts, qui sont des éléments clés des territoires villageois et essentiels pour garantir les moyens de subsistance des membres de la communauté. Ces politiques entravent les efforts des communautés locales pour gouverner leurs terres de façon globale, et en raison de leur position juridique désavantageuse, il leur est difficile de se protéger des groupes extérieurs, qu'il s'agisse d'organisations de conservation, de migrants saisonniers ou permanents, de sociétés minières ou d'acteurs de l'agriculture intensive, qui cherchent à obtenir des terres et des ressources dans ces espaces.

2. La pression exercée sur le gouvernement national par la communauté internationale de la conservation et les bailleurs de fonds pour établir des enclos de conservation a abouti à la mise en réserve de 7,1 millions d'hectares du territoire malgache dans des aires protégées, dont les deux tiers au cours des deux dernières décennies (Gardner et al. 2018). La plupart, sinon la totalité, des terres relevant des nouvelles aires protégées se trouvent dans les limites de territoires traditionnels établis (Aubert 2013). Ces enclos ont réduit les ressources foncières et forestières disponibles pour les communautés locales, souvent sans s'accompagner d'une augmentation parallèle de nouvelles opportunités de moyens de subsistance (Blanc-Pamard 2012).

3. Les politiques nationales de développement économique qui encouragent les acquisitions foncières à grande échelle pour les plantations destinées aux biocarburants (Medernach et Burnod 2013 ; Gingembre 2017), à l'agrobusiness (Rasoamalalavao 2011) et à l'exploitation minière (Seagle 2012) ont réduit les terres et les ressources disponibles pour les communautés rurales. Ces dernières années, les investisseurs extérieurs ont eu tendance à louer des terres que les responsables étatiques ou locaux considèrent comme " inoccupées », mais que les communautés locales utilisent depuis longtemps soit comme des pâturages pour le bétail, soit comme réserves foncières pour l'expansion agricole. Dans certaines régions, les villages voisins s'opposent les uns aux autres, les uns voyant dans l'exploitation minière et l'agrobusiness une source potentielle d'emplois à long terme, tandis que les autres s'opposent à la perte de terres nécessaires pour garantir leur subsistance (Medernach et Burnod 2013 ; Gingembre 2017).

4. Les vagues successives d'immigration, ainsi que la transformation économique induite, passant du pastoralisme à l'agriculture sédentaire, constituent un vecteur important des changements d'utilisation et de tenure des terres sur le plateau Mahafaly et la zone côtière adjacente (Moizo 2001) ainsi que dans la région du Menabe (Fauroux 2001). En effet, dans ces deux régions, l'afflux de migrants à la recherche de terres à cultiver s'est accompagné d'une déforestation accrue ${ }^{19}$ et d'une perturbation du mode de vie des pasteurs transhumants ${ }^{20}$. En parallèle, les changements structurels des économies locales ont permis aux migrants les plus aisés d'acquérir un plus grand pouvoir politique, affaiblissant ainsi le pouvoir et la légitimité apparente des autorités traditionnelles. Plusieurs facteurs ont contribué à l'augmentation de la migration, notamment la pénurie de terres dans les Hautes Terres du Centre (Poulain et Razanakoto 2014) ; la sécheresse prolongée dans le sud de Madagascar liée au changement climatique (IOM 2019); les politiques gouvernementales soutenant le développement de l'agrobusiness, avec une augmentation associée des besoins en main-d'œuvre salariée dans les zones relativement peu peuplées ; et l'émergence de solides marchés d'exportation pour certaines cultures, comme le maïs (Scales 2011).

19 L'étude de Jones et al. (2018:13) sur la migration vers les zones frontalières forestières dans l'est de Madagascar «n'a trouvé aucune preuve que les migrants étaient plus susceptibles d'avoir défriché des terres de la forêt ou d'avoir défriché une plus grande superficie de terres que les non-migrants ». Ils attribuent l'augmentation de la déforestation dans leur zone d'étude à l'augmentation de la demande associée à la migration intérieure plutôt qu'aux pratiques d'utilisation des terres faites par les migrants.

20 La transhumance est une forme de pastoralisme ou de nomadisme, un mouvement saisonnier du bétail entre des pâturages fixes d'été et d'hiver. 
5. La croissance démographique interne affecte également les systèmes de tenure dans de nombreuses communautés rurales. Madagascar connaît un taux de croissance démographique de $2,7 \%$, la majeure partie de l'augmentation se produisant dans les zones rurales (UN 2019). Dans de nombreuses régions des Hautes Terres du Centre, ainsi que dans certaines parties des Hautes Terres du Sud et sur le plateau Mahafaly, les terres qui conviennent à la culture du riz paddy sont complètement occupées, ce qui entraîne une pression sur les Hautes Terres (« tanety ») pour la culture du riz pluvial et d'autres cultures. À son tour, la demande en « tanety » a réduit le nombre de terres disponibles pour le pâturage, perturbant les moyens de subsistance des pasteurs.

6. L'évolution des normes socioculturelles affecte également les systèmes de tenure de manière bénéfique pour certains et préjudiciable pour d'autres. Les lois nationales qui accordent aux femmes des droits égaux à la terre permettent à certaines d'accéder plus facilement à la terre. Malgré leurs défauts, les processus de gestion participative des forêts ont ouvert des espaces où des membres de communautés auparavant marginalisés peuvent avoir la possibilité de faire entendre leur voix dans les décisions qui affectent leur accès à la terre et aux ressources. En revanche, à mesure que les normes évoluent, les systèmes traditionnels qui assuraient la protection des forêts tombent en désuétude dans certaines régions. Associées à un État corrompu et ayant peu d'autorité, les zones qui étaient autrefois gérées comme un bien commun et soumises à une réglementation, même limitée, sont devenues de facto en accès libre.

7. Le changement climatique qui a entraîné des conditions météorologiques plus extrêmes (c'est-à-dire des précipitations réduites et plus irrégulières, des inondations plus intenses et des températures extrêmes) exerce une pression sur des zones, comme le plateau d'Ihorombe, qui étaient déjà vulnérables aux inondations, à la sécheresse et aux criquets (Gingembre 2017). Dans le sud-ouest de Madagascar, la réduction des précipitations annuelles a réduit la quantité de fourrage disponible, exerçant une pression plus forte sur les zones où le fourrage samata est encore disponible (Goetter et Neudert 2016). Face aux pénuries prolongées de récoltes et à la diminution des réserves de fourrage pour le bétail, de nombreux habitants du sud de Madagascar ont migré, de manière temporaire ou permanente, vers des zones où les terres productives sont plus abondantes (Poulain et Razanakoto 2014) ${ }^{21}$.

\subsection{Les principales lacunes présentes dans la recherche}

Un nombre étonnamment élevé de publications, qu'il s'agisse de chapitres de rapports ethnographiques, de rapports techniques pour des organisations internationales ou pour le Gouvernement malgache, de livres ou d'articles publiés dans des revues scientifiques, abordent les systèmes de tenure coutumière à Madagascar. Bien que très utile, cette abondance de documents compte quelques lacunes en termes de couverture des systèmes de tenure, et particulièrement sur les parties du système qui sont gérées comme des ressources communales. Certains des domaines où la recherche semble être assez limitée sont décrits ci-dessous.

1. Disparités géographiques : Notre recherche a révélé que les études sur la tenure coutumière publiées depuis 1990, particulièrement celles relatives aux forêts, ont tendance à se concentrer dans les zones géographiques suivantes : le plateau Mahafaly et les plaines côtières associées, le corridor forestier de Ranomafana-Andringitra-Ivohibe (RAI), le corridor forestier de Mantadia-Zahamena, le plateau d'Ihorombe, la région du Menabe et les Hautes Terres du Centre (notamment autour du lac Alaotra et dans la région de Vakinankaratra). En comparaison, nous avons trouvé peu d'études provenant des régions du nord-ouest, du nord, du nord-est, du sud et du sud-est.

21 Les recherches menées par Canavesio en 2015 sur la migration dans le sud de Madagascar ont montré que le changement climatique n'est pas en soi la cause de l'émigration, mais qu'il agit plutôt comme un catalyseur de la migration dans un contexte où le système socio-économique est extrêmement fragile en raison de crises sociales, politiques et économiques chroniques. 
2. Disparités entre les groupes ethniques : Les systèmes de tenure coutumière des Antakarana, Bara, Betsileo, Betsimisaraka, Merina, Sakalava et Tanala étaient les plus représentés dans la littérature que nous avons étudiée. Bien que notre étude n'ait pas eu pour but d'être exhaustive, nous avons entrepris une recherche très approfondie de documents en anglais et en français postérieurs à 1990, sans trouver beaucoup d'études portant sur d'autres groupes ethniques.

3. Disparités de genre : Nous n'avons trouvé que quelques études qui allaient au-delà d'une description sommaire des droits fonciers et forestiers des femmes, ou de leur rôle dans les décisions de gouvernance forestière et foncière. En effet, la plupart des études qui ont fourni des analyses raisonnablement approfondies sur les droits et les rôles des femmes dans les systèmes de tenure coutumière ont été réalisées dans les années 1990 ou au début des années 2000. Il existe donc un besoin urgent d'études de cas approfondies sur les droits fonciers des femmes et de leur rôle dans la gouvernance des terres et des forêts partout dans le pays.

4. La tenure des pâturages dans le centre, le nord-ouest, le nord et l'est de Madagascar : La littérature sur la tenure des pâturages se concentre fortement sur les régions du sud et du sud-ouest de Madagascar. En revanche, très peu de recherches ont été effectuées sur la tenure coutumière des pâturages dans le nord, le nord-ouest, le nord-est, l'est et les Hautes Terres du Centre du pays. Dans la mesure où le bétail est économiquement et symboliquement important dans tout le pays, il s'agit d'un domaine où la recherche sur les systèmes de tenure pastorale s'avère plus que nécessaire.

5. Un panorama incomplet des éléments constitutifs des systèmes de tenure : Bien que de nombreuses études abordent certains aspects des systèmes de tenure coutumière à Madagascar, très peu fournissent une cartographie complète de la nature des droits, des personnes, des ressources, des périodes et des circonstances concernées. Ceci est particulièrement vrai pour les ressources forestières et les pâturages. La plupart des débats relatifs à la tenure forestière se concentrent sur les droits de défricher ou de couper des arbres, avec une attention moindre aux droits relatifs à d'autres produits ou ressources, comme les sources, les marais ou les lacs.

En plus de ces lacunes, les domaines thématiques que nous n'avons pas pu couvrir en détail, mais qui mériteraient davantage de recherches, incluent :

- Les systèmes agroforestiers ;

- Les programmes de reboisement et de restauration forestière ;

- La gouvernance foncière décentralisée et la planification de l'utilisation des terres (afin de mieux comprendre les interactions entre les systèmes de gouvernance foncière communale et traditionnelle) ;

- Les systèmes de cogestion des forêts et des aires protégées. 


\section{Conclusion}

« S'il est important que la réforme foncière soit renforcée, l'objectif n'est pas de promouvoir une formalisation obligatoire et systématique des droits de propriété individuels ou collectifs. L'objectif est d'insister sur la reconnaissance effective des droits locaux et sur la décentralisation de la gestion foncière, telle que définie dans les lois foncières de 2005, afin de protéger les droits des populations locales et, surtout, d'assurer la reconnaissance de leur pouvoir sur l'accès aux terres » (Burnod et al. 2013).

1. Notre étude démontre que bien que les systèmes de tenure coutumière à Madagascar partagent plusieurs caractéristiques communes importantes, il existe une hétérogénéité entre et au sein des groupes ethniques ainsi que sur le plan géographique. De fait, une approche unique pour la reconnaissance des droits de tenure coutumiers, y compris des autorités chargées d'attribuer les terres, s'avérerait probablement contre-productive. En effet, l'un des principaux enseignements tirés des expériences avec la loi GELOSE et la GCF est que l'utilisation d'un modèle uniformisé ou d'une formule toute faite est vouée à l'échec. De la même manière que l'on n'envisagerait pas de gérer toutes les forêts de Madagascar (qui comptent l'une des plus riches biodiversités du monde) en utilisant un modèle unique, la diversité des systèmes de tenure et des moyens de subsistance doit être reconnue et soutenue.

2. Notre étude montre également la présence d'une hétérogénéité dynamique dans les intérêts et un certain pouvoir, tant au sein des communautés qu'entre elles. Dans certaines régions, les propriétaires aisés de bétail ont intérêt à conserver les forêts, qu'ils utilisent pour le pâturage et comme abri pour leurs troupeaux, tandis que les membres de la communauté qui exploitent les zones situées sur les Hautes Terres de cette communauté ont intérêt à défricher les forêts pour accroître leur accès aux terres fertiles. Les habitants d'un hameau situé à proximité d'une forêt ont souvent une conception différente des règles qui devraient régir l'accès et l'utilisation de la forêt par rapport aux habitants de villages plus éloignés ou aux migrants saisonniers qui cherchent à vivre de l'exploitation forestière. Compte tenu de l'hétérogénéité des intérêts présents même au sein des petites communautés, ainsi que de la présence de déséquilibres de pouvoir, souvent anciens, au sein des communautés, ce serait une erreur de supposer que les anciens ou les autorités locales parlent toujours au nom de « la communauté ». En effet, il peut être difficile de trouver des personnes capables de représenter les intérêts d'une zone géographique aussi petite qu'un fokontany. Les anciens et autres autorités locales ne tiennent pas toujours compte de l'avis des autres membres moins puissants de la communauté, comme les femmes, les jeunes et les migrants, ou ils peuvent même prendre des décisions qui favorisent leurs propres intérêts au détriment de ceux de la communauté au sens large. C'est probablement la raison pour laquelle, dans certains cas de concessions foncières, les élites économiques qui n'avaient pas de pouvoir politique ont négocié des ententes avec les investisseurs (Medernach et Burnod 2013).

3. Lorsqu'on la relie à l'étude des textes juridiques, cette observation souligne la nécessité d'examiner attentivement quelles sont les institutions les plus appropriées pour régir les terres détenues par la communauté, dans l'hypothèse où des réformes foncières visant à reconnaître la tenure coutumière sur les espaces communs seraient mises en œuvre. Une grande partie du débat actuel sur la reconnaissance de la tenure coutumière se concentre sur le fokonolona comme étant l'institution la plus appropriée pour exercer une autorité sur les espaces communs locaux reconnus par l'État. Néanmoins, dans la plupart des régions, les fokonolona n'ont probablement jamais fonctionné comme des instances démocratiques. Notre étude documentaire a révélé qu'en général, les décisions sont prises par les chefs locaux ou un petit groupe de personnes, plutôt qu'à la suite d'un large consensus issu d'un processus délibératif inclusif. 
4. Les partisans de la réforme foncière partent du principe qu'une fois les droits des communautés sur leurs terres reconnus, les terres situées sur leurs territoires continueront d'être détenues et gérées au niveau communautaire. Pourtant, il se peut qu'une fois leurs revendications reconnues, les membres et chefs de certaines communautés décident de leur propre initiative d'octroyer des terres à leurs propres membres à titre privé, une pratique qui, selon notre étude documentaire, est assez fréquente dans les systèmes traditionnels actuels. En effet, l'espoir de pouvoir convertir la forêt en terres agricoles à un moment donné peut être un facteur de motivation pour certaines COBA.

5. Nous pensons qu'il serait prudent de reconnaître qu'il existe des différences significatives dans la capacité et la volonté politique des communautés à gérer leurs espaces communs. Sur la base de notre analyse, nous formulons les hypothèses suivantes.

Hypothèse 1 - Dans les zones où la cohésion sociale est élevée, les systèmes traditionnels restent largement fonctionnels et semblent fournir des cadres raisonnablement stables et efficaces grâce auxquels les droits d'accès, d'usage, de gestion et de transfert des terres ou des ressources sont attribués et appliqués, avec arbitrage des conflits.

Hypothèse 2 - Dans les zones où la cohésion sociale s'est rompue, souvent en raison d'une restructuration de l'économie rurale locale qui sape le pouvoir politique des autorités traditionnelles, les membres de la communauté et les personnes extérieures sont plus susceptibles d'avoir recours au système étatique. Dans la mesure où les deux systèmes sont déficients, on pourrait s'attendre à ce que les conflits soient plus nombreux dans ces régions.

Des recherches analysant plus en détail ces hypothèses s'avéreraient utiles pour cerner les éléments qui sont indispensables au bon fonctionnement des régimes fonciers coutumiers, pour savoir en quoi ils fonctionnent bien et pour quelles personnes (et qui sont les personnes désavantagées). Ces recherches pourraient permettre d'identifier les éléments du système qui doivent être réformés pour viser davantage d'inclusion et d'équité dans la répartition des avantages et des coûts.

6. Un dernier point important à souligner est que les institutions et la gouvernance locales sont profondément impactées par les relations existant entre les institutions étatiques et coutumières. Par exemple, bien que la loi GELOSE autorise le transfert des droits de gestion des pâturages, ainsi que ceux des forêts, on s'est peu intéressé jusqu'à présent à cet aspect de la loi. Comme souligné précédemment, étant donné l'importance sociale et économique du zébu dans la vie des Malgaches, il s'agit d'un aspect de la tenure coutumière qui mérite une plus grande attention.

Comme le montre cette analyse, l'impact positif des règles régissant les droits et l'utilisation des terres et des ressources sur les résultats en matière de conservation dépend du degré de légitimité de celles et ceux qui élaborent et appliquent les règles, ainsi que du fait que les règles reflètent ou non les réalités et les valeurs locales. Ceci plaide en faveur d'une reconnaissance légale claire des institutions traditionnelles locales et de leur autorité pour établir et réglementer l'utilisation des terres et des forêts. Néanmoins, les résultats des institutions traditionnelles locales doivent être au niveau des attentes du public quant à l'attribution équitable et à la gestion durable des droits fonciers et forestiers. Par conséquent, des formes appropriées de contrôle et de réglementation par les instances étatiques sont importantes et légitimes, à condition que les règles n'aient pas pour effet pervers de saper la capacité de la communauté à gérer efficacement les ressources. 


\section{References}

Andriamalala $\mathrm{G}$ et Gardner C. 2010. L'utilisation du dina comme outil de gouvernance des ressources naturelles: Leçons tirés de Velondriake, sud-ouest de Madagascar. Tropical Conservation Science 3(4):447-472.

Aubert, S, Rambintsaotra, S et Razafiarijaona, J. 2013. L'insécurité foncière dans et autour des Aires Protégées de Madagascar : un obstacle à surmonter pour la conservation de la biodiversité et le développement rural. Développement Durable et Territoires 4(1):1-24.

Barry LS, Bonte P, D’Onofrio S, Govoroff N, Jamard J-L, Mathieu N-C, Porqueres i Gené E, Wilgaux J, Zempléni A et Zonabend F. 2000. Glossaire de la parenté. L'Homme 154-155:721-732.

Bérard M-H. 2011. Légitimité des normes environnementales dans la gestion locale de la forêt à Madagascar. Canadian Journal of Law and Society 26(1):89-111.

Berger L. 2006. Ways of the ancestors, ways of development: The Ankaraña peoples facing globalization. Etudes Rurales 2(178):129-160.

Bidou J-E, Droy I et Fauroux E. 2008. Communes et regions à Madagascar: De nouveau acteurs dans la gestion locale de l'environnment. Mondes en Développement 141:29-46.

Blanc-Pamard C. 2012. La gestion contractualisée des forêts en pays Betsileo et Tanala (Madagascar): Un dispositive dyssymétrique. Cahiers d'Outre-Mer 257:47-82.

Blanc-Pamard C et Fauroux E. 2004. L'illusion participative: Exemples oust-malgaches. Autrepart 31:3-19.

Boué C et Colin J-P. 2018. Land certification as a substitute or complement to local procedures? Securing rural land transactions in the Malagasy highlands. Land Use Policy 72:192-200. https:// doi.org/10.1016/j.landusepol.2017.12.049

Brown, ML. 2004. Reclaiming lost ancestors and acknowledging slave descent: Insights from Madagascar. Comparative Studies in Society and History 46(3):616-645.

Burnod P, Gingembre M et Ratsialonana RA. 2013. Competition over authority and access: International land deals in Madagascar. Development and Change 44(2):357-379. https://doi. org/10.1111/dech. 12015

Canavesio R. 2015. Les migrations dans le sud de Madagascar: Entre sécheresses occasionnelles et crise socio-économique structurelle. Autrepart 2(74-75):259-278.

Covell M. 1987. Madagascar: Politics, Economics, and Society. London: Frances Pinter.

Cullman G. 2015. Community forest management as virtualism in northeastern Madagascar. Human Ecology 43:29-41.

Di Roberto, H. 2020. Le marché foncier, une affaire de famille? Une analyse institutionnelle des transactions de terres agricoles dans les Hautes Terres à Madagascar. Thèse du doctorat. France: Université de Montpellier.

Elmqvist T, Pyykönen M, Tengö M, Rakotondrasoa F, Rabakonandrianina E, Radimilahy C. 2007. Patterns of loss and regeneration of tropical dry forest in Madagascar: The social institutional context. PLoS ONE 2(5): e402. doi:10.1371/journal.pone.0000402

Evers S. 2006. Expropriated from the hereafter: The fate of the landless in the Southern Highlands of Madagascar. Journal of Peasant Studies 33(3):413-444 https://doi. org/10.1080/03066150601062928

Evers S. 2001. Constructing history, culture and inequality: The Betsileo in the extreme Southern Highlands of Madagascar. Thèse du doctorat. Amsterdam: Université d'Amsterdam.

Fauroux E. 2002. Les sociétés rurales de l'Ouest malgache: Des transformations profondes et complexes. Afrique Contemporaine 202-203:111-132.

Fauroux E. 2001. Dynamiques migratoires, tensions fonciers et deforestation dans l'Ouest malgache. Dans Razanaka S, Grouzis M, Milleville P, Moizo B et Aubry C. eds. Sociétés Paysannes, Transitions Agraires et Dynamiques Écologiques dans le Sud-Ouest de Madagascar. Antananarivo: CNRE/IRD, pp. 91-105. 
Feller C et Sandron F. 2019. Parcours de recherche à Madagascar: l'IRD-Orstom et ses partenaires. Marseilles: IRD.

Fritz-Vietta NVM, Röttger C et Stoll-Kleeman S. Community-based management in two biosphere reserves in Madagascar - distinctions and similarities: What can be learned from different approaches? Madagascar Conservation and Development 4(2):86-97.

Gardner, C, Nicoll ME, Birknshaw, C, Harris, A, Lewis, RE, Rakotomalala, D, et Ratsifandrihamanana, AN. 2018. The rapid expansion of Madagascar's protected area system. Biological Conservation 220:29-36.

Gezon L. 2002. Marriage, kin, and compensation: A socio-political ecology of gender in Ankarana, Madagascar. Anthropological Quarterly 75(4):675-706.

Gezon L. 1999. From adversary to son: Political and ecological process in northern Madagascar. Journal of Anthropological Research 55(1):71-97.

Gezon L. 1997. Political ecology and conflict in Ankarana, Madagascar. Ethnology 36(2):85-100.

Gingembre M. 2017. Being heard: Local people in negotiations over large-scale land deals. A case study from Madagascar. Thèse du doctorat. Brighton, UK: University of Sussex.

Goedefroit S. 1988. La société Sakalava du Menabe: Approche anthropologique d'un ensemble regional de Madagascar. Thèse du doctorat. Paris: Université de Paris I.

Goetter J et Neudert R. 2016. New rules are not rules: Privatization of pastoral commons and local attempts at curtailment in southwest Madagascar. International Journal of the Commons 10(2):617-64. https://doi.org/10.18352/ijc.743

Henkels D. 2002. A close-up of Malagasy environmental law. Vermont Journal of Environmental Law 3:1-16.

Horning N. 2004. The limits of rules: When rules promote forest conservation and when they do notInsights from Bara Country, Madagascar. Thèse du doctorat. New York: Université de Cornell.

International Organization for Migration (IOM). 2020. IOM Madagascar Annual report 2019. International Organization for Migration in Madagascar.

Jarosz L. 1991. Women as rice sharecroppers in Madagascar. Society and Natural Resources 4:53-63.

Jones PG, Mandimbiniaina R, Kelly R, Ranjatson P, Rakotojoelina B, Schreckenberg K et Poudyal M. 2018. Human migration to the forest frontier: Implications for land use change and conservation management. Geography and Environment. https://doi.org/10.1002/geo2.50

Karpe PM, Randrianarison M, Rambinintsaotra, S, et Aubert S. 2007. La pratique judiciaire dans le domaine foncier à Madagascar. Droit et Cultures 54:213-239.

Klein J, Réau B, Kalland I et Edwards M. 2007. Conservation, development, and a heterogeneous community: The case of Ambohitantely Special Reserve, Madagascar. Society and Natural Resources 20(5):451-467. https://doi.org/10.1080/08941920701211900

Kneitz P. 2016. Introduction: La paix du fihavanana. Dans Kneitz P. ed. Fihavanana - La Vision d'une Société Paisible à Madagascar: Perspectives Anthropologiques, Historiques Et Sociologiques. $2^{\text {ème }}$ édition. Halle an der Saale, Allemagne: Universitätsverlag Halle-Wittenberg, pp. 15-72.

Lambek, M et Walsh, A. 1997. The imagined community of the Antankarana: Identity, history, and ritual in northern Madagascar. Journal of Religion in Africa 27(3):308-333.

McConnell WJ. 2002. Misconstrued land use in Vohibazaha: Participatory planning in the periphery of Madagascar's Mantadia National Park. Land Use Policy 19:217-230.

McLain R, Ranjatson P, Lawry S, Rakotonirina JM, Randrianasolo R et Razafimbelo NT. 2019. Tenure challenges to implementing forest landscape restoration in northwestern Madagascar. CIFOR info brief no. 273. Bogor, Indonésie: CIFOR. https://www.cifor.org/library/7492

Medernach K et Burnod P. 2013. Recompositions inattendues d'un système agraire malgache par l'agrobusiness (Unexpected changes in a Madagascan agrarian system: The role of corporate agriculture). Études Rurales 191:63-76.

Messerli P. 2003. Alternatives à la culture sur brûlis sur la Falaise Est de Madagascar: Stratégies en vue d'une gestion plus durable des terres. African Studies Series A17. Thèse du doctorat. Bern, Allemagne: Université de Bern.

Moizo B. 2001. Controle de l'espace et enjeux fonciers en pays Bara: Les relations authochtonesmigrants. Dans Razanaka S, Grouzis M, Milleville P, Moizo B et Aubry C, eds. Sociétés Paysannes, Transitions Agraires et Dynamiques Écologiques dans le Sud-ouest de Madagascar. Antananarivo: CNRE/IRD, pp. 75-89. 
Muttenzer F. 2006. Déforestation et droit coutumier à Madagascar: L'historicité d'une politique foncière. Thèse du doctorat. Genève: Université de Genève.

Ottino P. 1998. Les champs de l'ancestralité à Madagascar: Parenté, alliance et patrimoine. Paris: Karthala et ORSTOM.

Pollini J. 2007. Slash-and-burn cultivation and deforestation in the Malagasy rain forests: Representations and realities. Thèse du doctorat. New York: Université de Cornell. p. 776.

Poulain M et Razanakoto T. 2014. Migration à Madagascar: Profil National 2013. Genève: OIM (Organisation Internationale pour les Migrations).

Raison J-P. 1991. Dynamismes ruraux et contrastes fonciers dans Madagascar en crise. Tiers-Monde 32(128):901-915.

Ranjatson P. 2011. La sécurisation des usages forestiers par les réseaux sociaux et l'économie: Deux exemples aux lisières de la reserve spéciale de Manongarivo et du corridor forestier Ranomafana Andringitra. Antananarivo, Madagascar: University of Antananarivo.

Ranjatson P, McLain R, Mananga J, Randrianasolo R, Razafimbelo T et Lawry S. 2019. Tenure security and forest landscape restoration: Results from exploratory research in Boeny, Madagascar. Présentation à la conférence de la Banque mondiale 2019 sur la terre et la pauvreté. Washington DC, 25-29 mars 2019.

Rasoamalalavao C, Ratovonjanahary R, Rasoamampionona C, Razafitody JF, Razafindrahasy A et Harimanga A. 2011. La décentralisation de la gestion foncière à Madagascar: Cas des communes rurales de Sahambavy et d'Analavory. IS Academy: Land Governance for Equitable and Sustainable Development. Netherlands: LANDAC.

Rosés M. 2016. Fihavanana local, fihavanana national: Les usages d'une coutume juridique locale à Madagascar. Dans Kneitz P ed. Fihavanana - La Vision d'une Société Paisible à Madagascar: Perspectives Anthropologiques, Historiques Et Sociologiques. $2^{\mathrm{ème}}$ édition. Halle an der Saale, Allemagne: Universitätsverlag Halle-Wittenberg, pp. 237-262.

Sandron F. 2016. Le fihavanana au quotidien. Dans Kneitz P ed. Fihavanana - La Vision d'une Société Paisible à Madagascar: Perspectives Anthropologiques, Historiques Et Sociologiques.

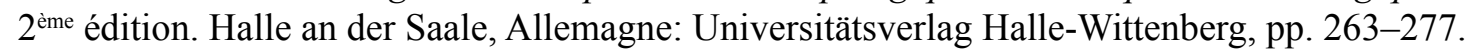

Scales IR. 2012. Lost in translation: Conflicting views of deforestation, land use and identity in western Madagascar. The Geographical Journal 178(1):67-79.

Scales IR. 2011. Farming at the forest frontier: Land use and landscape change in western Madagascar, 1896-2005. Environment and History 17(4):499-524.

Scheidecker G. 2016. Cattle, conflicts, and gendarmes in southern Madagascar: A local perspective on fihavanana gasy. Dans Kneitz P. ed. Fihavanana - La Vision d'une Société Paisible à

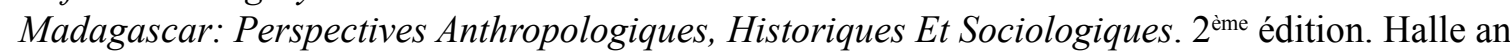
der Saale, Allemagne: Universitätsverlag Halle-Wittenberg, pp. 129-156.

Schlager E et Ostrom E. 1992. Property-rights regimes and natural resources: A conceptual analysis. Land Economics 68(3):249-262.

Seagle C. 2012. Inverting the impacts: Mining, conservation and sustainability claims near the Rio Tinto/QMM ilmenite mine in Southeast Madagascar. Journal of Peasant Studies 39(2):447-477. https://doi.org/10.1080/03066150.2012.671769

Serva M et Pasquini M. 2020. Dialects of Madagascar. PLoS ONE 15(10):e0240170. https://doi. org/10.1371/journal.pone.0240170

Sharp LA. 1993. The possessed and the dispossessed: Spirits, identity and power in a Madagascar migrant town. Berkeley: University of California Press. http://ark.cdlib.org/ark:/13030/ft6tlnb4hz/

Southall A. 1971. Ideology and group composition in Madagascar. American Anthropologist 73(1):144-164.

Spack S. 2005. Gestion environnementale décentralisée de la réserve spéciale de Manongarivo (NordOuest de Madagascar). Thèse du doctorat. Neuchâtel, Suisse: Université de Neuchâtel.

Tengö, M, Johansson, K, Rakotondrasoa, F, Lundberg, J, Andriamaherilala, JA, Elmqvist, T. 2007. Taboos and forest governance: Informal protection of hot spot dry forest in southern Madagascar. Ambio 36(8):683-691.

Thibaud B et Colin A. 2010. Tensions foncières et recompositions territoriales dans la basse vallée de l'Onilahy (Sud-Ouest Malgache). Annales de Géographie 6(676):697-709. 
Thielsen K. 2016. Which form of agreement works for community-based management? A case study from southwestern Madagascar. Madagascar Conservation \& Development 11(2):66-76. https:// doi.org/104314/mcd.v11i2.5

UN (United Nations). 2019. World Population Prospects 2019, Volume II: Demographic Profiles (ST/ESA/SER.A/427). United Nations, Department of Economic and Social Affairs, Population Division.

Valkonen AH. 2018. Conceptions, practices and power plays around the notion of tenure security: Examining the land policy development and implementation process in Madagascar. Thèse du doctorat. Université de East Anglia, School of International Development.

Walsh A. 2002. Responsibility, taboos and 'the freedom to do otherwise' in Ankarana, Northern Madagascar. Journal of the Royal Anthropological Institute 8(3):451-468.

Widman M et Hart R. 2019. Joint land titling and household bargaining in Madagascar. Feminist Economics 25(4):211-239 https://doi.org/10.1080/13545701.2019.1665195

World Bank. 2006. Madagascar: Land and Property Review. Washington DC: World Bank.

World Bank. 2004. Décentralisation à Madagascar. Washington DC: World Bank. http://documents. worldbank.org/curated/en/807221468773390434/Decentralisation-a-Madagascar 



\section{DOI: 10.17528/cifor/008388}

Les documents de travail du CIFOR contiennent des résultats préliminaires ou anticipés concernant d'importantes questions sur les forêts tropicales qui nécessitent d'être publiées à l'avance. Ces documents sont produits pour informer et inciter la discussion. Leur contenu a été revisé à l'interne, mais n'a pas subi la procédure d'examen par les pairs.

Le manque d'informations sur les systèmes coutumiers vivants qui gèrent les biens communs à Madagascar entrave les efforts visant à identifier les niveaux auxquels les collectivités chargées d'attribuer les terres et de faire respecter les revendications foncières devraient recevoir une reconnaissance légale. Pour aider à combler ce manque de connaissances et éclairer les réformes juridiques en cours visant à reconnaître la tenure collective, nous avons examiné les textes juridiques pertinents et les études de terrain des systèmes fonciers malgaches. Notre revue des textes juridiques a révélé que l'absence d'une définition juridique claire des collectivités situées au plus près des villageois ruraux est un obstacle majeur face aux efforts visant à reconnaître les droits d'attribution des terres et de gouvernance des institutions locales existantes. En même temps, notre revue des études de terrain sur les systèmes fonciers coutumiers démontre que ces systèmes continuent à fonctionner plus ou moins efficacement dans de nombreuses zones rurales de Madagascar. Les preuves suggèrent également que l'effet positif des systèmes de tenure coutumière sur les résultats de la conservation dépend du degré de légitimité perçue de ceux qui établissent et appliquent les règles, ainsi que du degré auquel les règles reflètent les réalités et les valeurs locales. Cela plaide en faveur d'une reconnaissance légale claire des institutions coutumières au niveau local, et de leur autorité pour établir et réglementer l'utilisation des terres et des forêts.

\begin{tabular}{|l|l|l|}
\hline & $\begin{array}{l}\text { Le programme de recherche du CGIAR sur les politiques, les institutions et les marchés (PIM) } \\
\text { a pour objet la recherche-action pour équiper les instances décisionnelles des informations }\end{array}$ \\
PROGRAM on & nécessaires à l'élaboration de politiques agricoles et alimentaires au service des intérêts \\
Policies, & des producteurs pauvres et des consommateurs, hommes et femmes. Le PIM s'appuie sur \\
Institutions, & les ressources des centres du CGIAR et de nombreux partenaires nationaux, régionaux et \\
and Markets & internationaux. Ce programme est dirigé par l'Institut International de Recherche sur les \\
Led by IFPRI & Politiques Alimentaires (IFPRI). www.pim.cgiar.org
\end{tabular}

\begin{tabular}{|l|l|}
\hline & Cette recherche a été menée par le CIFOR dans le cadre du Programme de Recherche du \\
& CGIAR sur les Forêts, les Arbres et l'Agroforesterie (FTA). Le FTA constitue le plus important \\
programme global de recherche pour le développement visant à amplifier la contribution des \\
forêts, des arbres et de l'agroforesterie au développement durable, à la sécurité alimentaire \\
et à la lutte contre le changement climatique. Le CIFOR dirige le FTA en partenariat avec \\
Bioversity International, le CATIE, le CIRAD, l'ICRAF, l'INBAR et TBI. \\
les Forêts, les Arbres et \\
l'Agroforesterie
\end{tabular}

\section{cifor.org | forestsnews.cifor.org}

Centre de recherche forestière internationale (CIFOR)

Le CIFOR contribue au bien-être humain, à l'équité et à l'intégrité de l'environnement en réalisant des travaux de recherche novateurs, en renforçant les capacités de ses partenaires et en nouant le dialogue avec tous les acteurs afin d'éclairer les politiques publiques et les pratiques qui touchent les forêts et les populations. Le CIFOR est un centre de recherche du CGIAR et dirige le Programme de recherche du CGIAR sur les forets, les arbres et l'agroforesterie (FTA). Le siège du CIFOR est à Bogor, Indonésie, avec des bureaux à Nairobi, Kénya ; Yaoundé, Cameroun ; Lima, Pérou et Bonn, Allemagne.

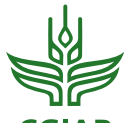

